\title{
PHASE TRANSITIONS AND METASTABILITY IN MARKOVIAN AND MOLECULAR SYSTEMS
}

\author{
By Wilhelm Huisinga, ${ }^{3}$ SeAn Meyn ${ }^{2}$ and Christof Schütte ${ }^{1}$ \\ Free University Berlin, University of Illinois and Free University Berlin
}

\begin{abstract}
Diffusion models arising in analysis of large biochemical models and other complex systems are typically far too complex for exact solution or even meaningful simulation. The purpose of this paper is to develop foundations for model reduction and new modeling techniques for diffusion models.

These foundations are all based upon the recent spectral theory of Markov processes. The main assumption imposed is $V$-uniform ergodicity of the process. This is equivalent to any common formulation of exponential ergodicity and is known to be far weaker than the Donsker-Varadahn conditions in large deviations theory. Under this assumption it is shown that the associated semigroup admits a spectral gap in a weighted $L_{\infty}$-norm and real eigenfunctions provide a decomposition of the state space into "almost"absorbing subsets. It is shown that the process mixes rapidly in each of these subsets prior to exiting and that the conditional distributions of exit times are approximately exponential.

These results represent a significant expansion of the classical WentzellFreidlin theory. In particular, the results require no special structure beyond geometric ergodicity; reversibility is not assumed and meaningful conclusions can be drawn even for models with significant variability.
\end{abstract}

\section{Contents}

1. Introduction

2. Spectral theory

2.1. Irreducible Markov process

2.2. Generators and spectra for Markov processes

2.3. Generators and spectra for nonprobabilistic semigroups

3. Metastability and exit rates

3.1. Exit rates

3.2. The twisted process

3.3. Consequences for exit times

3.4. Implications from large deviations theory

4. State space decompositions

4.1. Decompositions using a single eigenfunction

4.2. The shattered state space

Received February 2002; revised November 2002.

${ }^{1}$ Supported by the Deutsche Forschungsgesellschaft within SPP 1095.

${ }^{2}$ Supported in part by NSF Grant ECS 99-72957.

${ }^{3}$ Supported by the Deutsche Forschungsgesellschaft within the DFG Research Center "Mathematics for key technologies".

AMS 2000 subject classifications. 60F10, 60J25.

Key words and phrases. Markov process, large deviations. 
4.3. Error bounds for Markov chain approximations

5. Numerical example: the three-well potential

5.1. Exit rates and the shattered state space

5.2. Asymptotic behavior of eigensystem and Markov chain approximations

6. Outlook

1. Introduction. Markovian models are commonly used to represent the dynamics of a range of physical systems. In particular, diffusion models are a popular alternative to the classical description of molecular processes in terms of Hamiltonian equations of motion. Although these models may be faithful to physical realities, in practice a Markovian model is far too complex for exact solution or even long-term simulation. This is particularly true for biochemical systems with hundreds or thousands of atoms. How can we devise alternative models that capture essential features?

Recently there has been renewed interest in model reduction techniques based on variants of the classical Wentzell-Freidlin theory (see, e.g., [3, 6, 32]). The basic idea is that for certain Markov processes with small variability one can decompose the process into several "almost irreducible" subprocesses. To quantify this principle the recent paper [3] gives precise bounds on the distribution of exit times for certain countable state space chains and extensions to diffusions are contained in $[6,5,3]$. A key assumption imposed in these works is reversibility of the Markov process considered.

A related approach to the analysis of transition times is via the theory of quasistationary distributions of Markov process as introduced in $[37,41]$ for countable and general state-space processes, respectively. This theory has seen significant extensions in the recent papers [16, 17] through application of shift-coupling techniques [40]. These results are based upon the construction of an eigenfunction on a restricted domain of the state space. A similar approach is pursued in [11, 12] for diffusions with small noise to give bounds on exit times from a smooth domain. The papers $[34,36]$ describe new approaches to state space decomposition based on an analysis of the Perron cluster of eigenvalues for the full generator of the Markov processes. It is argued that eigenfunctions corresponding to dominant eigenvalues may be used to decompose the state space into metastable subsets.

The present paper builds upon the results and insights of the papers $[3,7$, 12, 16] and [34], combined with recent results concerning large deviations and spectral theory for $\psi$-irreducible Markov processes $[1,21,20]$. The main results demonstrate a strong form of quasi-stationarity for certain subsets of the state space. This implies precise bounds on the corresponding exit times and from these results we infer that the transition events of the diffusion are approximated by jump times of an associated continuous-time, finite state-space Markov chain.

A special case considered in [36] and in Section 5 is the Smoluchowski equation on $\mathbb{R}$,

$$
d X=-\frac{1}{\gamma} \nabla U(X) d t+\frac{\sigma}{\gamma} d W
$$




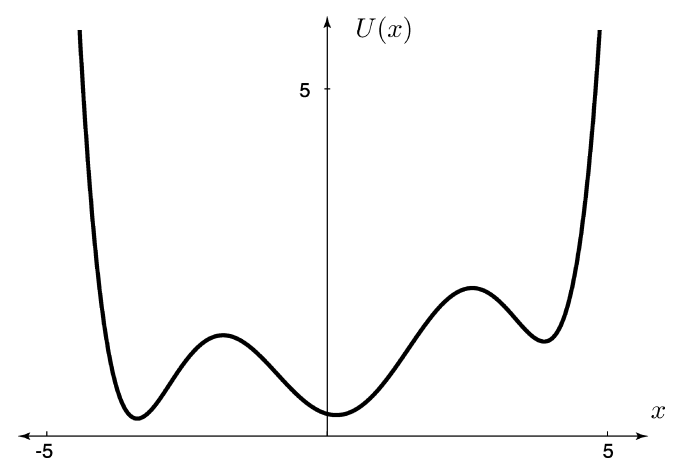

FIG. 1. The three-well potential $U(x)$.

for a given potential $U: \mathbb{R} \rightarrow \mathbb{R}_{+}$. The differential generator is defined for $h \in C^{2}$ by

$$
\mathscr{D} h(x)=\left(\frac{1}{2} \frac{\sigma^{2}}{\gamma^{2}} \Delta-\frac{1}{\gamma} \nabla U(x) \cdot \nabla\right) h .
$$

When $\sigma>0$ this is an elliptic diffusion, so that the semigroup has a family of smooth densities, $P^{t}(x, d y)=p(x, y ; t) d y, x, y \in \mathbb{R}^{d}$ [23]. Hence the Markov process $\mathbf{X}$ is $\psi$-irreducible, with $\psi$ equal to Lebesgue measure on $\mathbb{R}^{d}$.

A specific example is the three-well potential defined by the potential function $U$ shown in Figure 1. The function $U$ is a sixth-degree polynomial [see (32)]. For small $\sigma$, the process is almost decomposable into three processes, each attracted to a minimum of the function $U$.

In this paper we refine and extend these concepts for a general multivariate diffusion $\mathbf{X}$ by providing answers to the following questions:

(i) What is the appropriate function-analytic setting to investigate a spectral gap when the process is not reversible? When does the associated semigroup have a "spectral gap?"

(ii) It is well known that the value of the second eigenvalue determines the rate of convergence of the distributions for a Markov process. What is the physical significance of the associated second eigenfunction and higher-order eigenfunctions?

(iii) Can a complicated diffusion process be approximated by a simpler process, such as a finite state-space Markov chain, that preserves essential spectral structure and is a useful predictor of essential dynamics?

To address (i) we interpret the semigroup of the process as a semigroup of linear operators on a weighted $L_{\infty}$ space. We demonstrate in Theorem 3.1 that a small spectral gap in this setting is equivalent to a form of metastability of the state space. A spectral gap is also equivalent to geometric ergodicity, which is equivalent to the existence of a Lyapunov function [28, 9]. 
Under geometric ergodicity alone we demonstrate that real eigenfunctions provide a decomposition of the state space into metastable subsets. For any metastable set $M$ we construct a diffusion on $M$, the "twisted process," through a change of measure. We show that this restricted process is also geometrically ergodic, which implies that the original process mixes rapidly in each of these subsets prior to exiting.

To address approximations as in (iii) we consider the statistics of the exit time from a given metastable set $\mathrm{M}$. As a direct consequence of geometric ergodicity of the associated twisted process we find that the distribution of the exit time is approximately exponential. The magnitude of the error is related to the spectral gap for this twisted process.

The remainder of the paper is organized as follows. In the following section we review some ergodic theory from [9] and [21] and give a formal definition of spectrum for an ergodic diffusion. We also develop some structural theory for nonprobabilistic positive semigroups associated with the diffusion.

Section 3 introduces metastability and related concepts and develops structural results for the associated twisted process. Metastability is shown to be equivalent to geometric ergodicity of the twisted process, which gives a simple proof of the desired bounds on exit times. This section also contains a comparison of the results obtained here with conclusions from the large deviations theory of Wentzell and Freidlin.

The impact of a cluster of eigenvalues is investigated in Section 4 and in this section we describe a finite state-space approximating Markov chain. Section 5 contains a detailed numerical study of the Smoluchowski equation on $\mathbb{R}$ for the three-well potential.

2. Spectral theory. Here we review some general theory for $\psi$-irreducible Markov processes, including some recent spectral theory for the associated semigroup. The state space $\mathbb{X}$ is assumed to be an open, connected subset of $\mathbb{R}^{d}$ and we assume that time is continuous, $\mathbb{T}:=\mathbb{R}_{+}$. Eventually we will specialize to hypoelliptic diffusions on $\mathbb{X}$.

2.1. Irreducible Markov processes. Let $\psi$ denote a finite, positive measure on the Borel sigma-field $\mathscr{B}=\mathscr{B}(\mathbb{X})$ and let $\mathcal{B}^{+}$denote the set of functions $s: \mathbb{X} \rightarrow[0, \infty]$ satisfying $\psi(s)=\int s(x) \psi(d x)>0$. The set of finite, nonnegative measures $v$ satisfying $v(\mathbb{X})>0$ is denoted $\mathcal{M}^{+}$.

For each $\beta>0$ the resolvent kernel is given as the Laplace transform,

$$
R_{\beta}:=\int_{0}^{\infty} e^{-\beta t} P^{t} d t
$$

where $P^{t}$ is the transition function corresponding to the diffusion defined in (1). We note that $U_{\beta}:=\beta R_{\beta}$ is the transition kernel of the Markov chain on $\mathbb{X}$ obtained by sampling $\mathbf{X}$ at the jump times of a Poisson process. We write $R:=U_{\beta}=R_{\beta}$ when $\beta=1$. 


\section{Set characterizations.}

(i) A set $C \in \mathscr{B}$ is called full if $\psi\left(C^{c}\right)=0$.

(ii) The set $C \in \mathscr{B}$ is absorbing if $R\left(x, C^{c}\right)=0$ for $x \in C$. A nonempty absorbing set is always full ([28], Proposition 4.2.3).

(iii) A function $s$ and a measure $v$ are called small if, for some $\beta>0$,

$$
R_{\beta}(x, A) \geq s(x) v(A), \quad x \in \mathbb{X}, A \in \mathscr{B}(\mathbb{X}) .
$$

If $C \in \mathscr{B}$ and for some $\varepsilon>0$ the function $s:=\varepsilon \mathbf{1}_{C}$ is small, then we say that $C$ is small.

In Proposition 5.5.5 of [28] it is shown that for a $\psi$-irreducible process in discrete time, one can find a pair $(s, v)$ satisfying a bound analogous to (4) with $s(x)>0$ for all $x$, and with $v$ equivalent to the maximal irreducibility measure $\psi$ in the sense that they have the same null sets. This carries over to continuous time processes by considering the discrete-time Markov chain with transition kernel $R$ (see, e.g., [27]).

\section{Irreducibility and recurrence.}

(i) The Markov process $\mathbf{X}$ is called $\psi$-irreducible if

$$
R(x, s):=\int_{\mathbb{X}} R(x, d y) s(y)>0, \quad x \in \mathbb{X}, s \in \mathcal{B}^{+} .
$$

We assume that $\psi$ is maximal in the sense that $\psi^{\prime} \prec \psi$ for any other irreducibility measure $\psi^{\prime}$ [28].

(ii) $\mathbf{X}$ is called aperiodic if for any $s \in \mathcal{B}^{+}$, and any initial condition $x$,

$$
P^{t}(x, s)>0 \quad \text { for all } t \text { sufficiently large. }
$$

(iii) A $\psi$-irreducible Markov process is recurrent if

$$
\mathrm{E}_{x}\left[\int_{0}^{\infty} s(X(t)) d t\right]=\infty
$$

for all $s \in \mathscr{B}^{+}, x \in \mathbb{X}$.

(iv) A $\psi$-irreducible Markov process is Harris recurrent if

$$
\int_{0}^{\infty} s(X(t)) d t=\infty, \quad \text { a.s. }\left[\mathrm{P}_{x}\right]
$$

for all $s \in \mathscr{B}^{+}, x \in \mathbb{X}$.

For a given set $A \in \mathscr{B}$ we define the stopping times,

$$
\tau_{A}:=\inf \{t>0: X(t) \in A\}, \quad \rho_{A}:=\inf \left(t>0: \int_{0}^{t} \mathbf{1}(X(s) \in A) d s>0\right) .
$$

The stopping time $\tau_{A}$ is the usual first-hitting time and $\rho_{A}$ is the first time to enter the set $A$ for some non-null time interval. The use of the latter stopping time is 
to improve solidarity between the continuous time process and the Markov chain with transition kernel $R$. For example, we have

$$
\mathrm{P}_{x}\left(\rho_{A}<\infty\right)=0 \Longleftrightarrow R(x, A)=0, \quad x \in \mathbb{X}, A \in \mathscr{B} .
$$

Consequently, many of the characterizations given above may be conveniently expressed in terms of this stopping time, for example, a set $C \in \mathscr{B}(\mathbb{X})$ is absorbing if $\mathrm{P}_{x}\left(\rho_{C^{c}}<\infty\right)=0$ for all $x \in C$; and the process is Harris recurrent if $\mathrm{P}_{x}\left(\rho_{A}<\right.$ $\infty)=1$ for any $A \in \mathscr{B}^{+}$and all $x \in \mathbb{X}[29]$.

We henceforth restrict to a diffusion $\mathbf{X}=\{X(t): t \in \mathbb{T}\}$ evolving on $\mathbb{X}$, with differential generator given by

$$
\mathscr{D} h=\sum_{i} u_{i}(x) \frac{d}{d x_{i}} h(x)+\frac{1}{2} \sum_{i j} \Sigma_{i j}(x) \frac{d^{2}}{d x_{i} d x_{j}} h(x)
$$

or, in more compact notation,

$$
\mathscr{D}=u \cdot \nabla+\frac{1}{2} \operatorname{trace}(\Sigma \Delta) .
$$

We assume that the Markov process has continuous sample paths defined for all $t \geq 0$ for any initial condition-that is, the probability of finite escape is zero.

THEOREM 2.1. Suppose that $R$ is the resolvent kernel for the diffusion with generator given in (5), and suppose that the generator is hypoelliptic. Then $R$ is strong Feller and has a smooth density

$$
R(x, d y)=r(x, y) d y, \quad x, y \in \mathbb{X} .
$$

Suppose moreover that there is a state $x_{0} \in \mathbb{X}$ that is "reachable" in the following sense: For any $x \in \mathbb{X}$, and any open set $O$ whose closure contains $x_{0}$,

$$
P^{t}(x, O)>0 \quad \text { for all } t \in \mathbb{T} \text { sufficiently large. }
$$

Then, the Markov process is $\psi$-irreducible and aperiodic with $\psi(\cdot):=R\left(x_{0}, \cdot\right)$.

PROOF. This result together with a definition of hypoellipticity is given as [29], Theorem 3.3. The proof is based upon results from [22] and [23] and related results are obtained in $[25,38,39]$.

2.2. Generators and spectra for Markov processes. The ergodic theory and spectral theory described here are based upon the vector space setting developed in [28], Chapter 16. Let $V: \mathbb{X} \rightarrow[1, \infty)$ be a given function and denote by $L_{\infty}^{V}$ the vector space of measurable functions $h: \mathbb{X} \rightarrow \mathbb{C}$ satisfying

$$
\|h\|_{V}:=\sup _{x \in \mathbb{X}} \frac{|h(x)|}{V(x)}<\infty .
$$


The vector space $\mathcal{M}_{1}^{V}$ is the set of complex-valued measures $v$ on $\mathscr{B}$ such that

$$
\|v\|_{V}:=\int_{\mathbb{X}} V(x)|v(d x)|<\infty .
$$

For any kernel $\hat{P}$ on $\mathbb{X} \times \mathscr{B}$ the induced operator norm is defined by

$$
\|\hat{P}\|_{V}:=\sup \frac{\|\hat{P} h\|_{V}}{\|h\|_{V}}
$$

where the supremum is over $h \in L_{\infty}^{V},\|h\|_{V} \neq 0$. If $\hat{P}$ is a positive kernel [i.e., $\hat{P}(x, A) \geq 0$, for $x \in \mathbb{X}, A \in \mathscr{B}]$ and if for some $c<\infty$, we have $\hat{P} V \leq c V$, then $\hat{P}: L_{\infty}^{V} \rightarrow L_{\infty}^{V}$ is a bounded linear operator on $L_{\infty}^{V}$ and $\|\hat{P}\|_{V} \leq c$.

Several positive operators play a role in ergodic theory and spectral theory. The most important example is perhaps the bound (4), which means that the positive operator $R_{\beta}$ dominates the rank-one, positive operator $s \otimes v$ (" $\otimes$ " denotes the tensor product). The linear operator $[s \otimes v]: L_{\infty}^{V} \rightarrow L_{\infty}^{V}$ is necessarily bounded provided $R_{\beta}$ is bounded. In this case we have $s \in L_{\infty}^{V}$ and $v \in \mathcal{M}_{1}^{V}$. Moreover, when the resolvent kernel is a bounded linear operator we always have $R: L_{\infty}^{V} \rightarrow$ $C^{V}$, where

$$
C^{V}=\left\{g \in L_{\infty}^{V}:\left\|P^{t} g-g\right\|_{V} \rightarrow 0, t \downarrow 0\right\} .
$$

Formulations and characterizations of the spectral gap are facilitated by three different generators:

Generators.

(i) The extended generator $\mathcal{A}$ : We write $g=\mathcal{A} f$ if the adapted stochastic process $\left(M_{f}(t), \mathscr{F}_{t}\right)$ is a local martingale, where $\mathscr{F}_{t}=\sigma(X(s) ; 0 \leq s \leq t)$ and

$$
M_{f}(t):=f(X(t))-f(X(0))-\int_{0}^{t} g(X(s)) d s .
$$

(ii) The differential generator $\mathscr{D}$ : Defined on $C^{2}(\mathbb{X})$ via

$$
\mathscr{D} f=u \cdot \nabla f+\frac{1}{2} \operatorname{trace}(\Sigma \Delta f), \quad f \in C^{2} .
$$

(iii) The strong generator $\mathscr{D}_{V}$ : For a given $V: \mathbb{X} \rightarrow[1, \infty]$, finite a.e., we write $g=\mathscr{D}_{V} f$ if $f, g \in C^{V}$ and

$$
\left\|\frac{P^{t} f-f}{t}-g\right\|_{V} \rightarrow 0, \quad t \downarrow 0 .
$$

The extended generator $\mathcal{A}$ is a true extension of $\mathscr{D}$ in the sense that $\mathcal{A} f=\mathscr{D} f$ a.e. $[\psi]$ when $f \in C^{2}(\mathbb{X})$. Provided $R$ is a bounded linear operator on $L_{\infty}^{V}$, one can check that the domain of the strong generator is simply $\left\{R h: h \in C^{V}\right\}$ and that $\mathscr{D}_{V} R h=R h-h$ for any $h \in C^{V}$. The extended generator and differential generator are used in criteria for stability and to obtain bounds on the "essential spectrum" of the associated semigroup. The strong generator is used to define a spectral gap: 
Spectra and spectral gap. For a given $V: \mathbb{X} \rightarrow[1, \infty]$, finite a.e.:

(i) The spectrum $\mathrm{s}\left(\mathscr{D}_{V}\right)$ is the set of $\Lambda \in \mathbb{C}$ such that the inverse $\left[I \Lambda-\mathscr{D}_{V}\right]^{-1}$ does not exist as a bounded linear operator on $C^{V}$.

(ii) The generator admits a spectral gap if the set $\mathrm{s}\left(\mathscr{D}_{V}\right) \cap\{z \in \mathbb{C}: \operatorname{Re}(z) \geq-\varepsilon\}$ is finite for sufficiently small $\varepsilon>0$.

(iii) The Markov process is called $V$-uniformly ergodic if there is a spectral gap, $\{0\}=\mathrm{s}\left(\mathscr{D}_{V}\right) \cap\{z \in \mathbb{C}: \operatorname{Re}(z)=0\}$, and the eigenvalue $\Lambda=0$ is simple.

Theorem 5.2 of [9] provides the following consequences of $V$-uniform ergodicity:

THEOREM 2.2. If $\mathbf{X}$ is $V$-uniformly ergodic then:

(i) there is an invariant probability measure $\pi$, and the semigroup converges in norm:

$$
\left\|P^{t}-\mathbf{1} \otimes \pi\right\|_{V} \rightarrow 0 \quad \text { exponentially fast as } t \rightarrow \infty
$$

(ii) for any $B \in \mathcal{B}^{+}$, there exists $\bar{\Gamma}_{B}>0$ and $b<\infty$ such that

$$
\mathrm{P}_{x}\left\{\rho_{B} \geq t\right\} \leq b V(x) e^{-\bar{\Gamma}_{B} t}, \quad x \in \mathbb{X}, t \in \mathbb{T} .
$$

The following "drift condition" characterizes $V$-uniform ergodicity and is central to this paper. It is useful that we may use the extended generator and not the strong generator in (V4).

For constants $b<\infty$ and $\bar{\Gamma}>0$, a small function $s: \mathbb{X} \rightarrow[0, \infty)$, and (V4) a function $V: \mathbb{X} \rightarrow[1, \infty)$,

$$
\mathcal{A} V \leq-\bar{\Gamma} V+b s \text {. }
$$

Assumptions similar to (V4) are used in Donsker and Varadhan's classic papers (see $[42,8])$. It is shown in [20] that these assumptions actually imply that the diffusion has a discrete spectrum in the $V$-norm for some $V$ (see also [33]). Condition (V4) is equivalent only to a spectral gap and consequently it is a significantly weaker assumption.

THEOREM 2.3.

(i) Suppose that $\mathbf{X}$ is $\psi$-irreducible and aperiodic and suppose that (V4) holds for some $V: \mathbb{X} \rightarrow[1, \infty)$. Then $\mathbf{X}$ is $V$-uniformly ergodic.

(ii) Conversely, if the Markov process $\mathbf{X}$ is $V_{0}$-uniformly ergodic, then there exists a solution to (V4) with $V \in L_{\infty}^{V_{0}}$.

(iii) Suppose that the conditions of (i) hold, but the function $V: \mathbb{X} \rightarrow(0, \infty)$ is not known apriori to be bounded from below by 1 . If $\mathbf{X}$ is also recurrent then

$$
\varepsilon_{0}:=\inf _{x \in \mathbb{X}} V(x)>0,
$$

so that $\mathbf{X}$ is $V_{0}$-uniformly ergodic with $V_{0}:=\varepsilon_{0}^{-1} V$. 
PROOF. Theorem 2.3(i) and (ii) follow from Theorem 7.1 of [9].

To prove (iii) consider $U=\log (V)$ and apply the resolvent to obtain a bound of the form,

$$
R U \leq \log (R V) \leq U-\delta_{0}+b_{0} \mathbf{1}_{C},
$$

where $\delta_{0}>0 ; C=\left\{x: s(x) \geq \delta_{1}\right\} \in \mathcal{B}^{+}$for suitably small $\delta_{1}>0$ and $b_{0}<\infty$ is a constant. We then have, via the comparison theorem of [28],

$$
\delta_{0} \mathrm{E}_{x}\left[T_{C}\right] \leq U(x)+b_{0} \mathbf{1}_{C}(x),
$$

where $T_{C}$ denotes the first entrance-time to $C$ for the discrete-time Markov chain with transition law $R$. Here we have used recurrence of this Markov chain, which follows from the assumed recurrence of $\mathbf{X}$ [29]. We conclude that $\inf _{x \in \mathbb{X}} U(x)>$ $-b_{0}>-\infty$, which establishes (iii) with $\varepsilon_{0}:=e^{-b_{0}}$.

2.3. Generators and spectra for nonprobabilistic semigroups. For a given function $F: \mathbb{X} \rightarrow \mathbb{R} \cup\{\infty\}$ we consider the following positive semigroup,

$$
P_{F}^{t}(x, A)=\mathrm{E}_{x}\left[\mathbf{1}(X(t) \in A) \exp \left(-\int_{0}^{t} F(X(s)) d s\right)\right], \quad A \in \mathcal{B}, x \in \mathbb{X}, t \in \mathbb{T} .
$$

For any given $x, t$ the total mass $\lambda(x, t, \alpha)=P_{-\alpha F}^{t}(x, \mathbb{X}), \alpha \in \mathbb{R}$, is equal to the moment generating functions for the random variable $S_{t}=\int_{0}^{t} F(X(s)) d s$. This is a starting point for many papers concerning large deviations theory and risksensitive optimal control (see, e.g., [19, 2, 14, 13, 11]).

A strong generator can be defined in analogy with the probabilistic semigroup and we define the potential kernel associated with $\left\{P_{F}^{t}\right\}$ via

$$
R_{F}(x, A)=\int_{0}^{\infty} P_{F}^{t}(x, A) d t, \quad x \in \mathbb{X}, A \in \mathcal{B} .
$$

For example, when $F$ takes on the constant value $\beta$ for some positive $\beta \in \mathbb{R}_{+}$, then $R_{F}=R_{\beta}$. The following generalization of the resolvent equations are developed in [27] and [30].

THEOREM 2.4. For a given $F, G: \mathbb{X} \rightarrow \mathbb{R} \cup\{\infty\}$ we have the following:

(i) if $g: \mathbb{X} \rightarrow \mathbb{R}$ satisfies $R_{F}|g|(x)<\infty, x \in \mathbb{X}$, then

$$
\left[I_{F}-\mathcal{A}\right] R_{F} g=g
$$

(ii) if $G \geq F$ then the corresponding potential kernels are related by

$$
R_{F}=R_{G}+R_{G} I_{G-F} R_{F},
$$

where $I_{G-F}$ denotes the multiplication operator. 
For an arbitrary positive semigroup $\left\{\hat{P}^{t}\right\}$ the definitions of irreducibility, small sets and measures, and other set classifications remain the same in this nonprobabilistic setting. For a given function $V: \mathbb{X} \rightarrow[1, \infty]$, finite a.e., the spectral radius of $\left\{\hat{P}^{t}\right\}$ is given by

$$
\operatorname{sr}_{V}\left(\left\{\hat{P}^{t}\right\}\right):=\lim _{T \rightarrow \infty}\left(\left\|\hat{P}^{T}\right\|_{V}\right)^{1 / T} .
$$

Closely related is the Perron-Frobenius eigenvalue, defined for any small pair $(s, v)$ with $s \in \mathscr{B}^{+}, v \in \mathcal{M}^{+}$, via

$$
\operatorname{pfe}\left(\left\{\hat{P}^{t}\right\}\right):=\lim _{T \rightarrow \infty}\left(v \hat{P}^{T} s\right)^{1 / T} .
$$

The semigroup is called recurrent if

$$
\int_{0}^{\infty}\left(v \hat{P}^{t} s\right) e^{\Gamma t} d t=\infty
$$

where $\Gamma=-\log \left(\operatorname{pfe}\left(\left\{\hat{P}^{t}\right\}\right)\right)$; otherwise, it is called transient. A straightforward generalization of [31], Proposition 3.4, shows that these definitions are independent of the particular small pair chosen when the process is $\psi$-irreducible (note that [31] considers the convergence parameter, which is simply the reciprocal of the Perron-Frobenius eigenvalue). When $\hat{P}^{t}=P_{F}^{t}$ for some function $F$ on $\mathbb{X}$ we let $\operatorname{sr}_{V}(F), \operatorname{pfe}(F)$ denote the corresponding spectral radius and Perron-Frobenius eigenvalue.

Proposition 2.5(i) is a consequence of Proposition 3 of [20] and (ii) follows from Theorem 2.4(ii) with $F:=G-\gamma$.

Proposition 2.5. Suppose that $\mathbf{X}$ is a $\psi$-irreducible, hypoelliptic diffusion. Then:

(i) The functionals $\Gamma_{V}(F):=-\log \left(\operatorname{sr}_{V}(F)\right)$ and $\Gamma(F):=-\log (\operatorname{pfe}(F))$ are each concave on the domain $\{F: \mathbb{X} \rightarrow \mathbb{R} \cup\{\infty\}: \inf F(x)>-\infty\}$.

(ii) For a given function $G$ on $\mathbb{X}$, if $0<\gamma<\Gamma(G)$ then,

$$
\sum_{k=1}^{\infty} \gamma^{k-1} R_{G}^{k}=R_{G-\gamma}
$$

In analogy with $V$-uniform ergodicity, the semigroup $\left\{\hat{P}^{t}: t \in \mathbb{N}\right\}$ with generator $\widehat{D}_{V}$ is called $V$-uniform if the following conditions are satisfied:

(i) The constant $\Lambda:=-\Gamma_{V}(F)$ is a simple eigenvalue. That is, $\Lambda$ is an eigenvalue and the associated eigenspace is a one-dimensional subspace of $L_{\infty}^{V}$.

(ii) The generator admits spectral gap: for sufficiently small $\varepsilon>0$,

$$
\Lambda=\mathrm{s}\left(\widehat{D}_{V}\right) \cap\{z \in \mathbb{C}: \operatorname{Re}(z) \geq \Lambda-\varepsilon\} .
$$

We have the following analog of Theorem 2.3: 
THEOREM 2.6. Suppose that $\mathbf{X}$ is a $\psi$-irreducible, aperiodic, hypoelliptic diffusion. Let $F$ be a given function on $\mathbb{X}$ satisfying $\operatorname{pfe}(F)<\infty, \inf _{x \in \mathbb{X}} F(x)>$ $-\infty$ and suppose that the resolvent satisfies

$$
R_{F} V \leq \delta V+b s, \quad R_{F} \geq s \otimes v,
$$

where $V: \mathbb{X} \rightarrow[1, \infty]$ is finite a.e.; $0<\delta<\Gamma(F)^{-1} ; s: \mathbb{X} \rightarrow[0,1] ; b<\infty$; and $v$ is a probability measure on $\mathbb{X}$. Then, the semigroup $\left\{P_{F}^{t}\right\}$ is $V_{0}$-uniform for some $V_{0} \in L_{\infty}^{V}$.

ProOF. From Proposition 2.5(ii) we have for all $\gamma \in \mathbb{R}$ and all $B \in \mathcal{B}, x \in \mathbb{X}$,

$$
\sum_{k=1}^{\infty} \gamma^{k-1} R_{F}^{k}(x, B)=\mathrm{E}_{x}\left[\int_{0}^{\infty} \exp \left(-\int_{0}^{t} F(X(s)) d s\right) e^{\gamma t} \mathbf{1}\{X(t) \in B\} d t\right] .
$$

The right-hand side is finite whenever $\gamma<\Gamma(F)$ and the set $B$ is small. It follows that $\Gamma(F)^{-1}$ is the Perron-Frobenius eigenvalue for the discrete-time semigroup $\left\{R_{F}^{n}: n \geq 0\right\}$.

Let $h_{0}$ denote the Perron-Frobenius eigenfunction for $R_{F}$ given by

$$
h_{0}:=\sum_{k=0}^{\infty} \Gamma(F)^{k+1}\left(R_{F}-s \otimes v\right)^{k} s .
$$

We then have $R_{F} h_{0}=\Gamma(F)^{-1} h_{0}-\varepsilon s$, where $\varepsilon=1-v\left(h_{0}\right) \geq 0$ and $\varepsilon=0$ if and only if the semigroup $\left\{R_{F}^{k}: k \in \mathbb{N}\right\}$ is recurrent (see [31], Proposition 4.7).

Define the twisted kernel via

$$
\check{R}_{F}=\Gamma(F) I_{h_{0}}^{-1}\left[R_{F}+\varepsilon_{0} s \otimes v\right] I_{h_{0}},
$$

where $\varepsilon_{0}:=\varepsilon(1-\varepsilon)^{-1} \geq 0$ is chosen so that $\check{R}_{F}$ is a (probabilistic) transition kernel. Setting $\check{s}=\Gamma(F) \bar{h}_{0}^{-1} s$ and $\check{v}=v I_{h_{0}}$, we find that this is a small pair. In fact, $\check{R}_{F} \geq\left(1+\varepsilon_{0}\right) \check{s} \otimes \check{v}$ under our condition that $R_{F} \geq s \otimes v$. Moreover, one can check that $\sum_{k=0}^{\infty}\left(\check{v} \check{R}_{F}^{k} \check{s}\right)=\infty$, so that the semigroup $\left\{\check{R}_{F}^{k}: k \in \mathbb{N}\right\}$ is recurrent (see [31], Theorem 3.2). Setting $\check{V}=h_{0}^{-1} V$, we then have

$$
\check{R}_{F} \check{V}=\Gamma(F) h^{-1}\left[R_{F}+\varepsilon_{0} s \otimes \nu\right] V \leq h^{-1}[\delta \Gamma(F) V+b s] \leq \check{\delta} \check{V}+\check{b} \check{s},
$$

where $\check{b}<\infty$ and $\check{\delta}:=\delta \Gamma(F)<1$. Since the twisted kernel is recurrent, it then follows from Theorem 2.3(iii) that $\left\{\check{R}_{F}^{n}\right\}$ is $\check{V}$-uniform and that $\check{V}$ is uniformly bounded from below. This means that the inverse $\left[I z-\left(\check{R}_{F}-\left(1+\varepsilon_{0}\right) \check{S} \otimes \check{v}\right)\right]^{-1}$ exists in $\check{V}$-norm for all $z$ in a neighborhood of $z=1$. It immediately follows that $\left[I z-\left(R_{F}-s \otimes v\right)\right]^{-1}$ exists in $V$-norm for all $z$ in a neighborhood of $z=\Gamma(F)^{-1}$ and that $\left\{R_{F}^{n}\right\}$ is $V$-uniform (similar arguments are used in [21], Proposition 4.9). As in [9], Theorem 5.1, it then follows that the semigroup $\left\{P_{F}^{t}: t \in \mathbb{T}\right\}$ is $V_{0}$-uniform with $V_{0}=R_{F} V$. 
3. Metastability and exit rates. Much of the analysis here is based on the semigroups $\left\{P_{F}^{t}\right\}$ considered in Section 2.3 in the special case where $F=\infty \mathbf{1}_{A^{c}}$ for some $A \in \mathcal{B}$. When $F$ takes this form we denote the semigroup by $\left\{P_{A}^{t}\right\}$, which can be equivalently expressed,

$$
P_{A}^{t} g(x)=\mathrm{E}_{x}\left[g(X(t)) \mathbf{1}\left(\rho_{A^{c}} \geq t\right)\right], \quad g \in L_{\infty}, x \in \mathbb{X}, t \in \mathbb{T} .
$$

The associated potential kernel (8) is denoted $R_{A}$ when $F$ takes this form.

Let $\mathcal{C}$ denote the collection of all connected, open subsets of $\mathbb{X}$. For any $A, B \in \mathcal{C}$ we define

$$
A \circ B=\{\overline{A \cup B}\}^{\circ} .
$$

This is an open set and if $A \circ B \in \mathcal{C}$, we say that $A$ and $B$ are neighbors. The following assumptions will be imposed throughout this section. Theorem 2.1 provides readily verifiable conditions under which these assumptions are valid:

(i) The Markov process is an aperiodic, hypoelliptic diffusion, with continuous sample paths. The state space $\mathbb{X}$ is an open, connected subset of $\mathbb{R}^{d}$.

(ii) For each $A \in \mathcal{C}$ the semigroup $\left\{P_{A}^{t}\right\}$ is $\psi_{A}$-irreducible, where $\psi_{A}$ is Lebesgue measure restricted to $A$, and every compact subset of $A$ is a small set for $\left\{P_{A}^{t}\right\}$.

Eventually we will restrict to processes satisfying (V4).

3.1. Exit rates. Our goal in this section is to quantify the rate, at which the process moves between elements of $\mathcal{C}$. The motivation for the consideration of transition rates, rather than moments, is to set the stage for Markov chain approximations (see, e.g., Theorem 3.8).

\section{Exit rates and metastable sets.}

(i) The exit rate of $A \in \mathcal{C}$ is defined as $\Gamma(A):=-\log (\operatorname{pfe}(A))$, where $\operatorname{pfe}(A)$ denotes the Perron-Frobenius eigenvalue for the semigroup $\left\{P_{A}^{t}: t \in \mathbb{T}\right\}$ as defined in (11).

(ii) Given a pair of neighbors $A, B \in \mathcal{C}$, the exit rate from $A$ given $B$ is

$$
\Gamma(A \mid B):=\Gamma(A)-\Gamma(A \circ B) .
$$

(iii) A set $\mathrm{M} \in \mathcal{C}$ is called metastable with exit rate $\Gamma(\mathrm{M})$ if $\Gamma(A \mid \mathrm{M})>0$ for all $A \subset \mathrm{M}, A \neq \mathrm{M}, A \in \mathcal{C}$.

(iv) The $V$-exit rate of $A \in \mathcal{B}$ is given by $\Gamma_{V}(A):=-\log \left(\operatorname{sr}_{V}(A)\right)$, where $\operatorname{sr}_{V}(A)$ denotes the $V$-spectral radius of $\left\{P_{A}^{t}\right\}$.

(v) For $\mathrm{M} \in \mathcal{B}$ we say that $\mathrm{M}$ is $V$-metastable if $\Gamma_{V}(\mathrm{M})<\infty$ and

$$
\Gamma_{V}(A)>\Gamma_{V}(\mathrm{M})
$$

for any $A \subset \mathrm{M}$ satisfying $A \in \mathscr{B}$ and $\psi(\mathrm{M} / A)>0$. 
Metastability is closely related to $V$-uniform ergodicity. Here is one example:

THEOREM 3.1. If $\mathbf{X}$ is $V$-uniformly ergodic, then the state space $\mathbb{X}$ is $V$-metastable with exit rate equal to zero.

Proof. The $V$-norm ergodic theorem asserts that $P^{t} \rightarrow \mathbf{1} \otimes \pi$ in norm as $t \rightarrow \infty$. This implies that $\Gamma_{V}(\mathbb{X})=\Gamma(\mathbb{X})=0$. For any $A \in \mathscr{B}(\mathbb{X})$ with $B:=A^{c} \in$ $\mathcal{B}^{+}$, the bound (7) can be equivalently expressed,

$$
\left\|P_{A}^{t}\right\|_{V} \leq b e^{-\bar{\Gamma}_{B} t}, \quad t \in \mathbb{T} .
$$

From this we may conclude that $\Gamma_{V}(A) \geq \bar{\Gamma}_{B}>0$.

Since metastability is closely related to $V$-uniform ergodicity, it is natural that Lyapunov functions and eigenfunctions should play an important role in our analysis. To investigate the impact of Lyapunov functions we require the following restriction of the extended generator: For a set $A \in \mathcal{C}$ and functions $f, g: A \rightarrow \mathbb{R}$ we write " $g=\mathcal{A} f$ on $A$ " if $\left\{M_{f}\left(t \wedge \rho_{A^{c}}\right): t \in \mathbb{T}\right\}$ is a local martingale, where $M_{f}$ is given in (6).

THEOREM 3.2. Suppose that $\mathbf{X}$ is a diffusion satisfying (13). Then:

(i) For any set $A \in \mathcal{C}$, if there exists $0<\bar{\Gamma}<\infty$ and $h: A \rightarrow(0, \infty]$, finite almost everywhere, satisfying

$$
\mathcal{A} h \leq-\bar{\Gamma} h \quad \text { on } A,
$$

then $\Gamma(A) \geq \bar{\Gamma}$.

(ii) If $A \in \mathcal{C}$ with $0<\Gamma(A)<\infty$, then there exists $h: \mathbb{X} \rightarrow(0, \infty]$, finite almost everywhere, satisfying

$$
\mathcal{A} h \leq-\Gamma(A) h \quad \text { on } A .
$$

(iii) Suppose that $\mathrm{M} \in \mathcal{C}$ is $V$-metastable and its exit rate satisfies $\Gamma_{V}(\mathrm{M})=$ $\Gamma(\mathrm{M})<\infty$. Then there exists $h: \mathrm{M} \rightarrow(0, \infty)$ satisfying

$$
\mathcal{A} h=-\Gamma(\mathrm{M}) h \quad \text { on } \mathrm{M} \text {. }
$$

PROOF. If the conditions of (i) hold, then the stochastic process below is a local super-martingale,

$$
\bar{m}_{h}(t):=h(x)^{-1} h(X(t)) e^{\Gamma t} \mathbf{1}\left(\rho_{A^{c}} \geq t\right), \quad t \in \mathbb{T} .
$$

From Fatou's lemma we then have,

$$
\mathrm{E}\left[h(X(t)) \mathbf{1}\left(\rho_{A^{c}} \geq t\right)\right] \leq e^{-\bar{\Gamma} t} h(x), \quad x \in A .
$$


Let $(s, v)$ be any small pair satisfying $s \in \mathscr{B}^{+}, v \in \mathcal{M}^{+}$and

$$
s \leq h, \quad v(h)<\infty, \quad v\left(A^{c}\right)=0 .
$$

It immediately follows from the previous bound that for any $\Gamma<\bar{\Gamma}$,

$$
\int_{0}^{\infty} \mathrm{E}\left[s(X(t)) \mathbf{1}\left(\rho_{A^{c}} \geq t\right)\right] e^{\Gamma t} d t \leq \frac{h(x)}{\bar{\Gamma}-\Gamma}, \quad x \in A,
$$

from which we conclude that

$$
\int_{0}^{\infty}\left(\nu P_{A}^{t} s\right) e^{\Gamma t} d t \leq \frac{v(h)}{\bar{\Gamma}-\Gamma} .
$$

From the definitions it then follows that $\Gamma(A) \geq \Gamma$ and this proves (i) since $\Gamma<\bar{\Gamma}$ is arbitrary.

To see (ii) we first observe that the Perron-Frobenius eigenvalue for the discretetime semigroup $\left\{R_{A}^{n}\right\}$ is given by $\Gamma(A)^{-1}$ and a Perron-Frobenius eigenvector is then given by

$$
h_{0}(x)=\sum_{k=0}^{\infty} \Gamma(A)^{k+1}\left(R_{A}-s \otimes v\right)^{k} s(x),
$$

where $s, v$ is a small pair satisfying $R_{A} \geq s \otimes v$ (see the proof of Theorem 2.6). The function $h_{0}$ satisfies $v\left(h_{0}\right) \leq 1$, and consequently, for some $\varepsilon \geq 0$,

$$
R_{A} h_{0}=\Gamma(A)^{-1} h_{0}-\varepsilon s \leq \Gamma(A)^{-1} h_{0} .
$$

Letting $h=R_{A} h_{0}$, it then follows from Theorem 2.4 that

$$
\mathcal{A} h=\mathcal{A} R_{A} h_{0}=-h_{0}=-\Gamma(A)[h+\varepsilon s] \leq-\Gamma(A) h \quad \text { on } A .
$$

To see (iii) we construct a Lyapunov function $V_{\mathrm{M}}$ satisfying the conditions of Theorem 2.6. It then follows that the semigroup generated by the kernel $R_{\mathrm{M}}$ is recurrent, and that the constant $\varepsilon$ in (16) is zero. Consequently, this function $h$ solves the desired eigenfunction equation.

Fix $B \in \mathcal{C}$ with the following properties: $B \subset \mathrm{M} ; K:=\mathrm{M} \cap B^{c} \subset \mathrm{M}$ compact; $K \in \mathcal{B}^{+}$; and $\sup _{x \in K} V(x)<\infty$. Fix a pair $(s, \nu)$ satisfying $s \in \mathscr{B}^{+}, v \in \mathcal{M}^{+}$and $R_{B} \geq s \otimes v$. Then, for any $\gamma \leq \Gamma(B)$ we have the bound,

$$
\sum_{k=0}^{\infty} \gamma^{k+1} v\left[R_{B}-s \otimes v\right]^{k} s \leq 1
$$

Fix any such $\gamma$ satisfying $\Gamma(\mathrm{M})<\gamma<\Gamma(B)$. We then have, with $F_{\gamma}:=-\gamma+$ $\infty \mathbf{1}_{B^{c}}$,

$$
\sum_{k=1}^{\infty} \gamma^{k-1}\left[R_{B}-s \otimes \nu\right]^{k} V \leq \sum_{k=1}^{\infty} \gamma^{k-1} R_{B}^{k} V=R_{F_{\gamma}} V,
$$

and the right-hand side is in $L_{\infty}^{V}$ since $\gamma<\Gamma(B) \leq \Gamma_{V}(B)$. 
The measure $\mu_{B}$ given by

$$
\mu_{B}:=\sum_{k=0}^{\infty} \gamma^{k+1} v\left[R_{B}-s \otimes v\right]^{k}
$$

thus satisfies $\mu_{B}(V)<\infty$. Choose $g_{M}: M \rightarrow \mathbb{R}_{+}$continuous, satisfying $\mu_{B}\left(g_{M}\right)<$ $\infty$, and so that

$$
K_{n}=\left\{x \in \mathrm{M}: g_{\mathrm{M}}(x) \leq n V(x)\right\} \quad \text { is compact, } n \geq 1 .
$$

We then define

$$
V_{\mathrm{M}}:=\sum_{k=0}^{\infty} \gamma^{k+1}\left[R_{B}-s \otimes \nu\right]^{k} g_{\mathrm{M}},
$$

so that $b:=v\left(V_{\mathrm{M}}\right)=\mu_{B}\left(g_{\mathrm{M}}\right)<\infty$ and

$$
R_{B} V_{\mathrm{M}}=\left[R_{B}-s \otimes \nu\right] V_{\mathrm{M}}+[s \otimes \nu] V_{\mathrm{M}} \leq \gamma^{-1} V_{\mathrm{M}}+b s .
$$

The following is a generalization of the resolvent equation given in (10):

$$
R_{\mathrm{M}}=R_{B}+Q_{B} I_{\mathrm{M} \cap B^{c}} R_{\mathrm{M}},
$$

where $Q_{B}(x, d y):=\mathrm{P}_{x}\left(X\left(\rho_{B^{c}}\right) \in d y\right)$. Since $V_{\mathrm{M}}$ is bounded on $\mathrm{M} \cap B^{c}$, we then arrive at a bound of the form,

$$
R_{\mathrm{M}} V_{\mathrm{M}} \leq \gamma^{-1} V_{\mathrm{M}}+b_{\mathrm{M}},
$$

where $b_{\mathrm{M}}<\infty$ is constant.

Finally, for any $\delta_{0}$ satisfying $\Gamma(\mathrm{M})^{-1}>\delta_{0}>\gamma^{-1}$ we can find $b_{0}<\infty, n_{0}<\infty$ satisfying,

$$
R_{\mathrm{M}} V_{\mathrm{M}} \leq \delta_{0} V_{\mathrm{M}}+b_{0} \mathbf{1}_{K_{n_{0}}} .
$$

The set $K_{n_{0}}$ is a compact subset of $\mathrm{M}$ and, hence, also small for $R_{\mathrm{M}}$ under (13). An application of Theorem 2.6 then shows that the semigroup $\left\{R_{\mathrm{M}}^{n}: n \geq 0\right\}$ is $V_{\mathrm{M}}$-uniform, and that a solution to (14) exists with $h \in L_{\infty}^{V_{\mathrm{M}}}$.

3.2. The twisted process. In this section we investigate the consequences of the following "eigenfunction equation." Theorem 3.2(iii) provides evidence that (18) will typically hold when $M$ is metastable, and in this case we may take $\Gamma_{0}=\Gamma(\mathrm{M})$. Recall that $\mathscr{D}$ is the differential generator given in (5).

For some set $\mathrm{M} \in \mathcal{C}$, a function $h: \mathbb{X} \rightarrow \mathbb{R}$ that is $C^{2}$ in a neighborhood of the set $\overline{\mathrm{M}}$, and some $\Gamma_{0}<\infty$,

$$
h(x)>0 \quad \text { and } \quad \mathscr{D} h(x)=-\Gamma_{0} h(x) \quad \text { for } x \in \mathrm{M} .
$$

Under (18), for any $x \in \mathbb{X}$ the stochastic process

$$
m_{h}(t):=h(x)^{-1} h(X(t)) e^{\Gamma_{0} t}, \quad t \in \mathbb{T},
$$


is a positive martingale up to the stopping time $T_{\bullet}:=\rho_{\mathrm{M}^{c}}$. Hence it may serve in a change of measure in the following construction:

The twisted process is the Markov process $\check{\mathbf{X}}$ with state space $\mathrm{M}$ whose

semigroup is defined for any $g \in L_{\infty}(\mathrm{M})$ and any $x \in \mathrm{M}$ via

$$
\check{\mathrm{E}}_{x}[g(\check{X}(t))]:=\mathrm{E}_{x}\left[m_{h}(t) g(X(t)) \mathbf{1}\left(T_{\bullet}>t\right)\right] .
$$

The Markov process $\check{\mathbf{X}}$ is a diffusion, evolving on $\check{\mathbb{X}}:=\mathrm{M}$. The associated "twisted generator" is given in Proposition 3.4. Similar constructions are used in many recent references (see, e.g., $[10,21]$ ). What is unusual here is that the likelihood function defined using $h$ restricts the process to a proper subset of $\mathbb{X}$ (see also [16]).

Proposition 3.4 expresses the differential generator for the twisted process in terms of $\mathfrak{D}$. This representation is a consequence of the following lemma, whose proof is immediate from Ito's rule.

LEMmA 3.3. Suppose that $g, h: O \rightarrow \mathbb{R}$ are $C^{2}$ on the open set $O \subseteq \mathbb{X}$. The following identities hold on the set $O$ :

$$
\begin{aligned}
\mathscr{D} g h & =g \mathscr{D} h+h \mathscr{D} g+\langle\Sigma \nabla h, \nabla g\rangle, \\
\mathscr{D} h & =\left[\mathscr{D} H+\frac{1}{2} \nabla H^{T} \Sigma \nabla H\right] h,
\end{aligned}
$$

where in the second identity we assume that $h>0$ on $O$ and let $H$ denote its logarithm.

Proposition 3.4. Suppose that (13) and (18) hold. Then:

(i) The expectation operator $\check{\mathrm{E}}$ defines a diffusion on $\mathrm{M}$, up to the exit time $T_{\bullet}$. The differential generator is given by

$$
\check{\mathscr{D}}=I_{h^{-1}} \mathscr{D} I_{h}+\Gamma_{0} I=\mathscr{D}+\langle\Sigma(\nabla H), \nabla\rangle,
$$

where $I_{h}$ is the multiplication operator: $I_{h} g=h \cdot g$, and $H(x)=\log (h(x))$ for $x \in \mathrm{M}$.

(ii) The differential generator $\check{D}$ has the same diffusion coefficients as the original process and the generator $\check{D}$ is self adjoint whenever $\mathscr{D}$ is (with a new inner product weighted by $h$ ).

(iii) If $\mathbf{X}$ is a Smoluchowski equation on $\mathbb{X}$ with potential $U$ and if $\Sigma=\sigma^{2} I$ is independent of $x$, then $\check{\mathcal{D}}$ is the differential generator for a Smoluchowski equation with potential $U_{+}=U-\sigma^{2} H$.

PROOF. It is enough to establish (20), which is immediate from Lemma 3.3 and the eigenvector equation $\mathscr{D} h=-\Gamma h$ :

$$
\mathscr{D} g h=h\left(-\Gamma g+\mathscr{D} g+\left\langle\Sigma\left(h^{-1} \nabla h\right), \nabla g\right\rangle\right) .
$$


Multiplying both sides by $h^{-1}$ and adding $\Gamma g$ gives the required identity.

The following two results characterize metastability in terms of geometric ergodicity of the twisted process.

THEOREM 3.5. Assume that (13) and (18) hold. Suppose moreover that $\mathrm{M}$ is $V$-metastable, its exit rate satisfies $\Gamma_{0}=\Gamma_{V}(\mathrm{M})=\Gamma(\mathrm{M})$, and that the escape-time $T_{\bullet}$ for the twisted process is infinite a.s. Then the twisted process is $\breve{V}$-uniformly ergodic for some $\check{V}$ satisfying $h^{-1} \in L_{\infty}^{\check{V}}$.

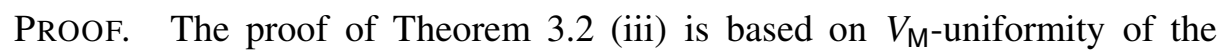
discrete-time semigroup generated by $R_{\mathrm{M}}$ [see definition (17)]. It follows that the discrete-time Markov chain with transition kernel

$$
\check{R}_{\mathrm{M}}:=I_{h}^{-1} R_{\mathrm{M}} I_{h}+\Gamma(\mathrm{M})^{-1}
$$

is $\check{V}$-uniformly ergodic with $\check{V}=h^{-1} V_{\mathrm{M}}$. This transition kernel is in fact a resolvent kernel for the twisted process defined (19). The result is then immediate from [9], Theorem 5.1, and our assumption that the escape-time for the twisted process is infinite a.s.

Theorem 3.6 provides a partial converse.

THEOREM 3.6. Assume that (13) and (18) hold. Suppose, moreover, that the escape-time for the twisted process is infinite a.s. and that the twisted process is $\check{V}$-uniformly ergodic for some $\check{V}: \mathrm{M} \rightarrow[1, \infty)$, with $h^{-1} \in L_{\infty}^{\check{V}}$. Then, the set $\mathrm{M}$ is both metastable and $V_{0}$-metastable, with common exit rate $\Gamma(\mathrm{M})=\Gamma_{V_{0}}(\mathrm{M})=\Gamma_{0}$ given in (18) and with $V_{0}=\check{V} h$.

PROOF. This is a consequence of the following representation: For any stopping time $\tau$ satisfying $\tau \leq T_{\bullet}$,

$$
\check{\mathrm{E}}_{x}\left[\int_{0}^{\tau} e^{\delta t} h^{-1}(\check{X}(t)) d t\right]=h^{-1}(x) \mathrm{E}_{x}\left[\int_{0}^{\tau} e^{\left(\delta+\Gamma_{0}\right) t} d t\right] .
$$

Let $A \subset \mathrm{M}$ satisfy $\psi\left(A^{c}\right)>0$ and let $\tau=\rho_{A^{c}}$. From $\check{V}$-uniformity of the twisted process and the assumption that $h^{-1} \in L_{\infty}^{\check{V}}$, there exists $\delta=\delta(A)>0$ and $b_{0}<\infty$ such that the left-hand side is bounded by $b_{0} \check{V}(x)$ [cf. (7)]. It follows that the $V_{0}$-exit rate from $A$ is bounded from below by $\Gamma_{0}+\delta(A)$.

It remains to show that the exit rate from $M$ is equal to $\Gamma_{0}$. This follows from the previous reasoning: Setting $\tau=T_{\bullet}$ gives

$$
\begin{aligned}
\infty & =\check{\mathrm{E}}_{x}\left[\int_{0}^{\infty} h^{-1}(\check{X}(t)) d t\right] \\
& =\check{\mathrm{E}}_{x}\left[\int_{0}^{T_{\bullet}} h^{-1}(\check{X}(t)) d t\right]=h^{-1}(x) \mathrm{E}_{x}\left[\int_{0}^{T_{\bullet}} e^{\Gamma_{0} t} d t\right] .
\end{aligned}
$$


Hence $\Gamma(M) \leq \Gamma_{0}$, but Theorem 3.2(i) already implies the reverse inequality.

We now provide more readily verifiable sufficient conditions under which the conclusions of Theorem 3.6 will hold.

THEOREM 3.7. Assume that (13) and (18) hold and that (V4) is also satisfied for a continuous function $V: \mathbb{X} \rightarrow[1, \infty)$. Suppose, moreover, that the Lyapunov function $V$ and the eigenfunction $h$ satisfy the following conditions:

(a) the constant $\Gamma_{0}$ in (18) satisfies $0<\Gamma_{0}<\bar{\Gamma}$;

(b) $h(x)>0$ for all $x \in \mathrm{M}$ and $h(x)=0$ for $x \in \partial \mathrm{M}:=\overline{\mathrm{M}} \backslash \mathrm{M}$;

(c) $(\nabla h(x))^{T} \Sigma(x)(\nabla h(x))>0$ for all $x \in \partial \mathrm{M}$;

(d) $K_{n}:=\{x \in \mathbb{X}: V(x) \leq n h(x)\}$ is a compact subset of $\mathbb{X}$ for all $n \geq 1$.

Then:

(i) The escape-time from $\mathrm{M}$ for the twisted process is infinite a.s. for $\check{X}(0)=$ $x \in \mathrm{M}$.

(ii) The twisted process is $\check{V}_{1}$-uniformly ergodic with $\check{V}_{1}=V / h$.

(iii) The set $\mathrm{M}$ is both metastable and $V$-metastable, with exit rate $\Gamma(\mathrm{M})=$ $\Gamma_{V}(\mathrm{M})=\Gamma_{0}$, where $\Gamma_{0}$ is given in (18).

PROOF. We consider the Markov process with twisted generator $\check{D}$ given in (20). For a given $0<\alpha<1$ write

$$
\check{V}_{1}:=h^{-1} V, \quad \check{V}_{2}:=h^{-1} h^{\alpha} \quad \text { and } \quad \check{V}:=\check{V}_{1}+\check{V}_{2} .
$$

Then from Lemma 3.3, (V4) and the eigenvector equation (18),

$$
\begin{aligned}
& \check{\mathscr{D}} \check{V}_{1}=\left[I_{h}^{-1} \mathscr{D} I_{h}+\Gamma_{0} I\right] h^{-1} V \\
& =h^{-1}\left[\mathscr{D}+\Gamma_{0}\right] V \\
& \leq-\left(\bar{\Gamma}-\Gamma_{0}\right) \check{V}_{1}+b h^{-1} s, \\
& \check{\mathscr{D}} \check{V}_{2}=\left[I_{h}^{-1} \mathscr{D} I_{h}+\Gamma_{0} I\right] h^{-1+\alpha} \\
& =h^{-1}\left[\mathscr{D} h^{\alpha}+\Gamma_{0} h^{\alpha}\right] \\
& =h^{\alpha-1}\left[\alpha \mathcal{D} H+\frac{1}{2} \alpha^{2} \nabla H^{T} \Sigma \nabla H+\Gamma_{0}\right] \\
& =h^{\alpha-1}\left[\alpha\left(h^{-1} \mathfrak{D} h-\frac{1}{2} \nabla H^{T} \Sigma \nabla H\right)+\frac{1}{2} \alpha^{2} \nabla H^{T} \Sigma \nabla H+\Gamma_{0}\right] \\
& =(1-\alpha) h^{\alpha-1}\left[\Gamma_{0}-\frac{1}{2} \alpha \nabla H^{T} \Sigma \nabla H\right],
\end{aligned}
$$

where the third and fourth identities follow from Lemma 3.3. Combining these bounds/equalities gives

$$
\begin{aligned}
\check{\mathscr{D}} \check{V} \leq & -\frac{1}{2}\left(\bar{\Gamma}-\Gamma_{0}\right) \check{V} \\
& +h^{\alpha-1}\left[(1-\alpha) \Gamma_{0}+\frac{1}{2}\left(\bar{\Gamma}-\Gamma_{0}\right)+b h^{-\alpha} s\right. \\
& \left.\quad-\frac{1}{2}\left(\bar{\Gamma}-\Gamma_{0}\right) V h^{-\alpha}-\frac{1}{2} h^{-2} \alpha(1-\alpha) \nabla h^{T} \Sigma \nabla h\right]
\end{aligned}
$$


where in the last line we have used the identity $\nabla H=h^{-1} \nabla h$.

Consequently, under assumption (a) we have the following version of (V4) for the twisted process: for sufficiently large $n_{0}$,

$$
\check{D} \check{V} \leq-\frac{1}{2}\left(\bar{\Gamma}-\Gamma_{0}\right) \check{V}+b_{0} \mathbf{1}_{K_{n_{0}}},
$$

where $b_{0}<\infty$, and $K_{n_{0}}$ given in (d) is compact. The drift condition (21) implies that $T_{\bullet}=\infty$ a.s. since $\check{V}$ has compact sublevel sets in M (see [29]). This proves (i) and (ii) is a consequence of the drift inequality (21), a form of (V4), which implies that $\check{\mathbf{X}}$ is $\check{V}$-uniformly ergodic. It is also $\check{V}_{1}$-uniformly ergodic with $\check{V}_{1}=V / h$ since $h \in L_{\infty}^{V}$ by assumption.

Part (iii) then follows from the foregoing conclusions and Theorem 3.6.

3.3. Consequences for exit times. We show here that $\check{V}$-uniform ergodicity of the twisted process implies strong distributional bounds on the exit time from a metastable set $\mathrm{M}$. The exit time $T_{\bullet}:=\rho_{\mathrm{M}^{c}}$ is approximately exponentially distributed, with rate $\Gamma(\mathrm{M})$, provided there is a sufficient spectral gap. Related approximations in a general setting were obtained recently in [17]. These bounds provide a bridge between the results established here and the large deviation theory of Wentzell and Freidlin. To make this precise we first review the standard characterizations of exponential random variables.

If the random variable $\rho$ is exponentially distributed with rate $\Gamma$, then the conditional distribution function and the conditional moment generating function for the residual life are given by

$$
\begin{array}{cl}
F(s)=\mathrm{P}[(\rho-T) \geq s \mid \rho \geq T]=e^{-\Gamma s}, & s \geq 0, T \geq 0 ; \\
M(\beta)=\mathrm{E}[\exp (\beta(\rho-T)) \mid \rho \geq T]=\frac{\Gamma}{\Gamma-\beta}, & \beta \leq \Gamma .
\end{array}
$$

These quantities are independent of $T$ only for exponential random variables. However, we show in Theorem 3.8 that these identities almost hold for the exit time $T_{\bullet}$ from a metastable set.

For the random variable $T_{\bullet}$ we again define the conditional distribution function and the conditional moment generating function for the residual life at time $T$ by

$$
\begin{aligned}
& F_{x}(s, T)=\mathrm{P}_{x}\left[\left(T_{\bullet}-T\right) \geq s \mid T_{\bullet} \geq T\right], \quad s \geq 0, T \geq 0 ; \\
& M_{x}(\beta, T)=\mathrm{E}_{x}\left[\exp \left(\beta\left(T_{\bullet}-T\right)\right) \mid T_{\bullet} \geq T\right], \quad \beta \leq \Gamma, T \geq 0 .
\end{aligned}
$$

Theorem 3.8 states that the rate of decay of the exit time is basically independent of the starting point. We note that the constant $\delta_{0}>0$ describes the rate of convergence of the distributions of the twisted process [cf. (24)] and this is 
precisely the spectral gap for the twisted process.

THEOREM 3.8. Suppose that the conditions of Theorem 3.7 hold, so that the set $\mathrm{M}$ is $V$-metastable with exit-rate $\Gamma>0$. Then there exists $\delta_{0}>0$ such that for all $s, T>0$ and all $\beta<\Gamma$,

$$
\begin{aligned}
F_{x}(s, T) & =e^{-\Gamma s}\left[\frac{1+O\left(e^{-\delta_{0} s} V(x) h^{-1}(x)\right)}{1+O\left(e^{-(T+s) \delta_{0}} V(x) h^{-1}(x)\right)}\right], \\
M_{x}(\beta, T) & =\frac{\Gamma}{\Gamma-\beta}+O\left(e^{-\delta_{0} T} V(x) h^{-1}(x)\right) .
\end{aligned}
$$

PROOF. From the definition of the twisted process we have for all $s \geq 0$ and all $x \in \mathrm{M}$,

$$
\begin{aligned}
\check{P}^{s} h^{-1}(x) & =\check{\mathrm{E}}_{x}\left[h^{-1}(\check{X}(s)) \mathbf{1}\left(s \leq T_{\bullet}\right)\right] \\
& =h^{-1}(x) \mathrm{E}_{x}\left[h(X(s)) h^{-1}(X(s)) e^{\Gamma s} \mathbf{1}\left(s \leq T_{\bullet}\right)\right] \\
& =h^{-1}(x) e^{\Gamma s} \mathrm{P}\left(s \leq T_{\bullet}\right) .
\end{aligned}
$$

An application of Theorem 3.7 implies that the twisted process is $\check{V}$-uniformly ergodic for some $\breve{V}$ satisfying $h^{-1} \in L_{\infty}^{\check{V}}$. It follows that we also have the following bound, for some $\delta_{0}>0$,

$$
\check{P}^{s} h^{-1}(x)=\check{\pi}\left(h^{-1}\right)+O\left(e^{-\delta_{0} s} V(x) h^{-1}(x)\right), \quad s \geq 0, x \in \mathbb{X} .
$$

This combined with (23) gives the bound

$$
e^{\Gamma s} \mathrm{P}_{x}\left\{s \leq T_{\bullet}\right\}=\left(\check{\pi}\left(h^{-1}\right) h(x)+O\left(e^{-\delta_{0} s} V(x)\right)\right)
$$

and this easily proves the result.

3.4. Implications from large deviations theory. We conclude this section with a comparison of our conclusions with those of Wentzell and Freidlin [15].

Consider some stable equilibrium point $x_{0}$ of the unperturbed ODE,

$$
d x=-\frac{1}{\gamma} \nabla U(x) d t
$$

and let $O$ be a region of attraction. We assume that $O$ is a (possibly semiinfinite) interval $O=\left(x_{a}, x_{b}\right) \subset \mathbb{R}$ containing $x_{0}$ and satisfying the following assumptions:

All trajectories of the deterministic ODE (25) starting at some point $x \in \bar{O}$ converge to $x_{0}$ as $t \rightarrow \infty$. Hence, there are neither other extrema nor limit cycles in $\bar{O}$.

Let $\tau_{O^{c}}(\sigma)$ denote the exit time of $O$ for the Smoluchowski process $\mathbf{X}$ defined by (1). Futhermore, denote $U_{0}:=U\left(x_{0}\right), U_{a}:=U\left(x_{a}\right), U_{b}:=U\left(x_{b}\right)$, and let

$$
U_{\text {bar }}:=\min \left\{U_{a}-U_{0}, U_{b}-U_{0}\right\}
$$




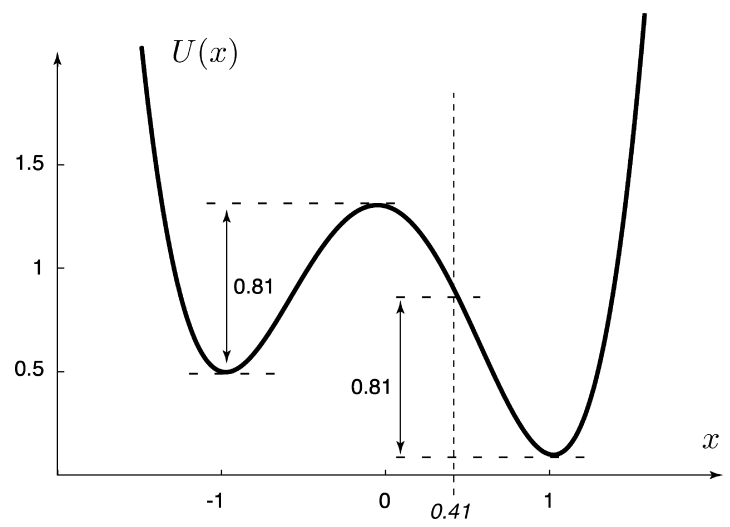

FIG. 2. The two-well potential $U(x)$. The barrier on the left $U_{\mathrm{bar}}=0.81$ defines a decomposition of the state space into two metastable subsets $\mathbb{X}=(-\infty, 0.41) \circ(0.41, \infty)$ with equal potential barrier $U_{\mathrm{bar}}=0.81$.

denote the minimal potential barrier the process must cross to leave $O$ when starting at $x_{0} \in O$.

The following result follows from [15] and [35]. It gives a simple bound on the exit rate from $O$ in terms of the smallest potential barrier one has to cross when leaving $O$.

THEOREM 3.9. Under the above assumption on $O$ we have the following bound on the exit time from $O$, for any initial condition $x_{0} \in O$,

$$
\lim _{\sigma \rightarrow 0} \sigma^{2} \log \mathrm{E}_{x_{0}}\left[\tau_{O^{c}}(\sigma)\right]=2 \gamma U_{\mathrm{bar}} .
$$

To illustrate Theorem 3.9 we examine the double-well potential shown in Figure 2. Denote the left local minimum of $U$ by $U\left(x_{l}\right)=U_{l}$, the right minimum by $U\left(x_{r}\right)=U_{r}$ and the local maximum by $U\left(x_{m}\right)=U_{m}$. In this example we have $U_{l}=0.50, U_{m}=1.31$ and $U_{r}=0.10$.

We wish to obtain bounds on the exit rates for the two open connected components $O_{\text {left }}=\left\{x<x_{m}\right\}$ and $O_{\text {right }}=\left\{x>x_{m}\right\}$, corresponding to the left and the right well, respectively. To fulfill the assumption that saddle-points are excluded we reduce these sets slightly and instead take $O_{\text {left }}=\left\{x<x_{m}-\varepsilon\right\}$ and $O_{\text {right }}=\left\{x>x_{m}+\varepsilon\right\}$ for some arbitrary small $\varepsilon>0$. An application of Theorem 3.9 yields the following conclusions:

(i) for $O_{\text {left }}$ we have $U_{\mathrm{bar}}=U_{m}-U_{l}=0.81+O\left(\varepsilon^{2}\right)$ and, hence,

$$
\lim _{\sigma \rightarrow 0} \sigma^{2} \log \mathrm{E}_{x}\left[\tau_{O_{\mathrm{left}}}(\sigma)\right] \approx 2 \gamma 0.81
$$

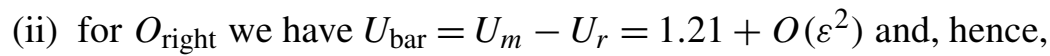

$$
\lim _{\sigma \rightarrow 0} \sigma^{2} \log \mathrm{E}_{x}\left[\tau_{O_{\text {right }}}(\sigma)\right] \approx 2 \gamma 1.21
$$


We now compare these conclusions with the results of the present paper. Applying Theorem 3.7 to the double-well potential we find that the second eigenfunction decomposes the state space into the two open components $\{a, b\} \subset$ $\mathcal{C}$ separated by the zero $z$ of $h_{2}$. What can we say about the asymptotics of $z$ for vanishing $\sigma$ ?

(i) A natural first guess is that $z$ approximates the saddle point of the potential. However, as discussed above, the Wentzell-Freidlin theory predicts that the distribution of exit times is a function of the minimal potential barrier the process has to cross in order to leave a given subset. Consequently, these rates are different for the two subsets $\mathrm{a}$ and $\mathrm{b}$ when $\sigma$ is small, which contradicts the fact that $\Gamma(\mathrm{a})=\Gamma(\mathrm{b})=\Gamma_{2}$.

We conclude that $z$ cannot approximate the saddle point of the potential as $\sigma \rightarrow 0$.

(ii) An alternative is the point $z$ on the the right-hand side of the saddle point defined by the condition that the minimal potential barrier $U_{\text {bar }}$ to exit from either of the two subsets is the same (see Figure 2). An extension of the Wentzell-Freidlin theory (see, e.g., [3, 4]) states that the asymptotic rates of both exit times are the same, which is in agreement with Theorems 3.7 and 3.8.

In view of (ii) we modify the subsets slightly so that $\widehat{O}_{\text {right }}=\{x<0.41\}$ and $\widehat{O}_{\text {left }}=\{x>0.41\}$. We thus obtain identical asymptotes for $\mathrm{E}_{x}\left[\tau_{\widehat{O}_{\text {right }}}(\sigma)\right]$ and $\mathrm{E}_{x}\left[\tau \widehat{\widehat{O}}_{\text {right }}(\sigma)\right]$ as $\sigma \rightarrow 0$. Figure 3 shows that the zero of $h_{2}$ does indeed approach the value 0.41 for vanishing $\sigma$. (Note in revision. Discussion in the recent paper [5] suggests that there is a strong relationship between saddle points of the potential
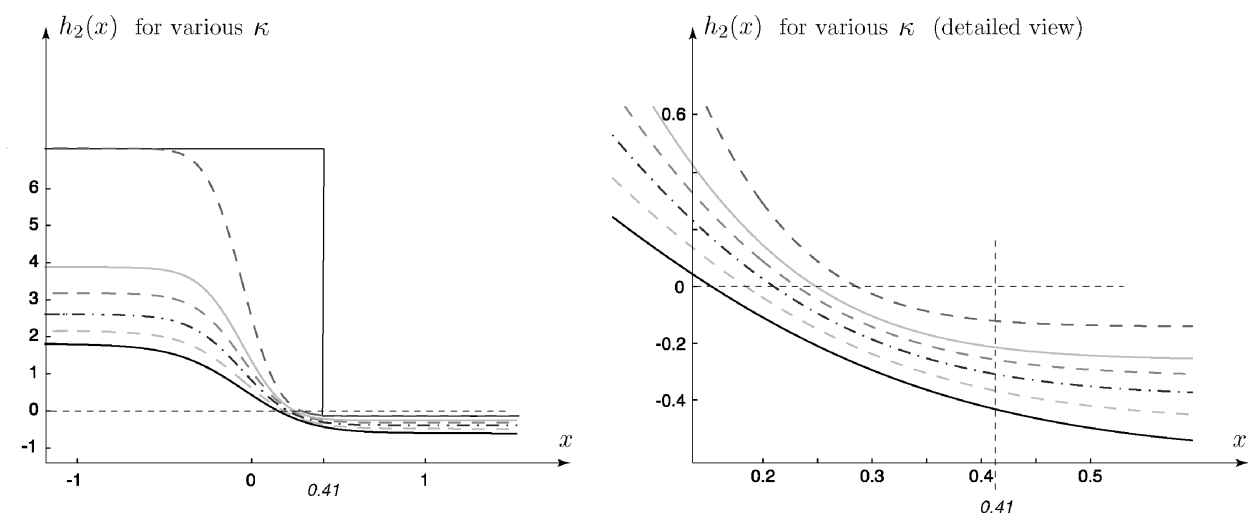

FIG. 3. At left is shown the second eigenfunction $h_{2}$ for the diffusion defined by the two-well potential for a range of values of the inverse temperature, $\kappa=2 \gamma / \sigma^{2}$, from $\kappa=1.5$ (solid line) to $\kappa=6.5$, (dashed line). The right-hand side shows a close-up for $x$ near the respective zeros. The zero moves towards the value 0.41 as $\kappa \uparrow \infty$. The step function shown at left with discontinuity at $x=0.41$ is a candidate limit of $h_{2}^{\kappa}$. 
function and structure of the eigenfunction for small $\kappa$.)

4. State space decompositions. In the previous section we considered in some detail the structure of a diffusion restricted to a single metastable set. In particular, Theorems 3.2, 3.6 and 3.7 provide characterizations of metastability in terms of an eigenfunction defined on the set $\mathrm{M}$. Here we obtain decompositions of the entire state space into metastable sets by considering eigenfunctions $h \in L_{\infty}^{V}$ for the generator $\mathscr{D}$ defined on all of $\mathbb{X}$. Under appropriate conditions, including $V$-uniform ergodicity, this provides a natural decomposition of the state space into metastable subsets.

4.1. Decompositions using a single eigenfunction. Let $h$ be a $C^{2}$ eigenfunction satisfying $\mathscr{D} h=\Lambda h$ and let $\left\{\mathrm{M}_{i}\right\} \subset \mathcal{C}$ denote the open, connected components of $\{x: h(x) \neq 0\}$. Fix any $i$ and assume without loss of generality that $h>0$ on $\mathrm{M}_{i}$ (otherwise, replace $h$ by $-h$ ). It follows that the desired eigenfunction equation holds

$$
\mathscr{D} h=-\Gamma h, \quad h>0, \text { on } \mathrm{M}_{i},
$$

where $\Gamma=|\Lambda|>0$. Under the conditions of Theorem 3.7 we can conclude that $\Gamma\left(\mathrm{M}_{i}\right) \geq \Gamma$ and that $\mathrm{M}_{i}$ is $V$-metastable.

To illustrate this decomposition consider the simplest diffusion: the onedimensional Gauss-Markov process with differential generator,

$$
\mathscr{D}=-a x \frac{d}{d x}+\frac{1}{2} \sigma^{2} \frac{d^{2}}{d x^{2}} .
$$

This is of the form (1) with potential function $U(x)=\frac{1}{2} a x^{2}$. We assume that $a>0$, so that the process is $V$-uniformly ergodic with $V(x)=\cosh (x), x \in \mathbb{R}$.

It is easily seen that $\left\{\Lambda_{k}=-(k-1) a: k=1,2, \ldots\right\}$ belongs to the spectrum of $\mathscr{D}_{V}$ (see, e.g., [24]) with associated eigenfunctions,

$$
\begin{aligned}
h_{1} & \equiv 1, & h_{2}(x) & =x, \\
h_{3}(x) & =\frac{1}{2} x^{2}-\frac{\sigma^{2}}{4 a}, & h_{4}(x) & =\frac{1}{3} x^{3}-\frac{\sigma^{2}}{2 a} x .
\end{aligned}
$$

The second eigenfunction $h_{2}$ decomposes the state space into the two sets $\mathbb{X}=M_{1} \circ M_{2}:=(-\infty, 0) \circ(0, \infty)$. The conditions of Theorem 3.7 are satisfied and consequently, each of the twisted processes on $\mathrm{M}_{1}$ or $\mathrm{M}_{2}$ is $\check{V}$-uniformly ergodic with $\check{V}=V / h_{2}=e^{x} /|x|, x \in \mathrm{M}_{i}, i=1,2$. Moreover, each of these sets is $V$-metastable with exit rate $\Gamma\left(\mathrm{M}_{i}\right)=a$. The twisted differential generator for the process on $\mathrm{M}_{2}$ is given by

$$
\begin{aligned}
\check{D}_{2} & =\mathscr{D}+\sigma^{2} H_{2}^{\prime} d x \\
& =\left(-a x+\sigma^{2} / x\right) d x+\frac{1}{2} \sigma^{2} d x^{2} .
\end{aligned}
$$


This is the generator for the Smoluchowski equation with potential $\frac{1}{2} a x^{2}-$ $\sigma^{2} \log (x), x \in \mathrm{M}_{2}$. One can check directly that this diffusion on $\mathrm{M}_{2}$ is $\check{V}$-uniformly ergodic.

Not all "twistings" give rise to an ergodic diffusion: applying the differential generator to the function $f(x):=\exp \left(\frac{1}{2} \beta x^{2}\right)$ with $\beta=2 a / \sigma^{2}$ gives

$$
\begin{aligned}
& \mathscr{D} f=-a \beta x^{2} f(x)+\frac{1}{2} \sigma^{2}\left(\beta+\beta^{2} x^{2}\right) f(x) \\
& =\left\{\frac{1}{2} \sigma^{2} \beta+\left(\frac{1}{2} \sigma^{2} \beta^{2}-a \beta\right) x^{2}\right\} f(x) \\
& =a f(x) \text {. }
\end{aligned}
$$

That is, the constant $a>0$ is a (generalized) eigenvalue for the differential generator $\mathscr{D}$ and $f$ is the corresponding (generalized) eigenfunction. Although $\mathbf{X}$ is a $V$-uniformly ergodic Markov process we see here that the (generalized) eigenvalue is equal to $a>\Gamma(\mathbb{X})=0$. Nevertheless, one can perform a transformation using $f$ to form a new diffusion on $\mathbb{R}$ via (20). The twisted process is a driftless Brownian motion on $\mathbb{R}$.

This shows that care must be taken in interpreting (generalized) eigenfunction equations for $\mathcal{D}$. In general, the inequality $\Gamma(\mathrm{M}) \geq \bar{\Gamma}$ in Theorem 3.2(i) may be strict and the twisted process may not be ergodic.

4.2. The shattered state space. If the connected components $\left\{\mathrm{M}_{i}\right\} \subset \mathcal{C}$ of $\left\{h_{2}(x) \neq 0\right\}$ are each metastable, then Theorem 3.8 suggests that the "indicator process" giving the current metastable set that the process resides should approximate a finite state-space Markov chain. However, the conclusions of Theorem 3.8 are not very meaningful unless the twisted process admits a significant spectral gap. Consequently, if the semigroup has a cluster of eigenvalues near zero, then an approximation by a finite state-space chain is not possible without performing a refined decomposition that takes into account the interaction of a cluster of eigenvalues.

Suppose that (V4) holds, fix $n \geq 2$, and suppose that $\left\{\Lambda_{i}: 1 \leq i \leq n+1\right\} \subset$ $(-\bar{\Gamma}, 0)$ are the $n+1$ first eigenvalues satisfying

$$
\begin{gathered}
\mathrm{s}\left(\mathscr{D}_{V}\right) \cap\{z \in \mathbb{C}: \operatorname{Re}(z)>-\bar{\Gamma}\} \\
=\left\{\Lambda_{i}: 1 \leq i \leq n+1\right\} .
\end{gathered}
$$

We assume the eigenvalues are distinct, real and ordered,

$$
\bar{\Gamma}>\left|\Lambda_{n+1}\right|>\left|\Lambda_{n}\right|>\cdots>\left|\Lambda_{1}\right|=0,
$$

and that $\left|\Lambda_{n+1} / \Lambda_{n}\right| \gg 1$.

For simplicity we take $n=3$ and we assume that each of the first four eigenvalues are simple. Hence, for each $i=1,2,3,4$ there exists a one-dimensional eigenspace spanned by an eigenfunction $h_{i} \in L_{\infty}^{V}$. An illustration of the assumed 

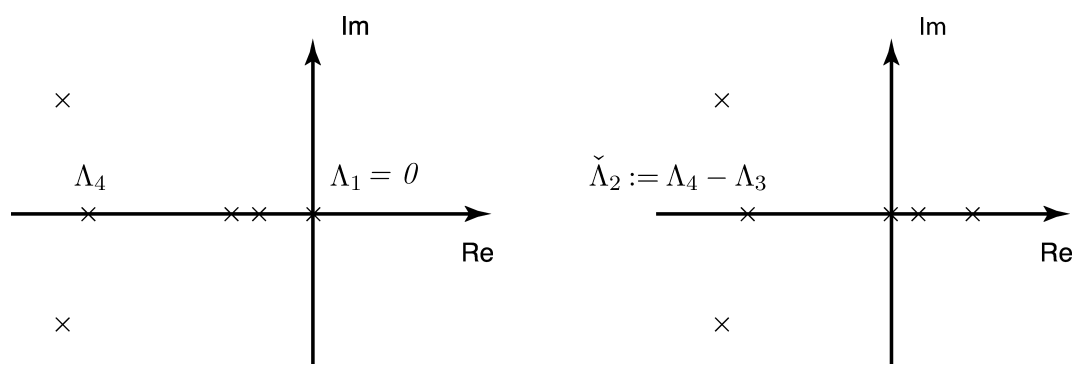

FIG. 4. At left is shown the spectrum of the generator $\mathfrak{D}_{V}$ and at right is shown the spectrum of $\check{D}$, viewed as a differential operator, where the third eigenfunction and eigenvalue are used to construct the twisted generator. The spectra shown in the right-half plane does not correspond to eigenfunctions in $L_{\infty}^{\check{V}}$.

eigenvalue structure is shown in Figure 4. We assume moreover that the conditions of Theorem 3.7 hold, so that in particular,

$$
\nabla h_{i}(x) \neq 0 \quad \text { whenever } h_{i}(x)=0, i=2,3,4 \text {. }
$$

For $m=2,3,4$, let $\left\{\mathrm{M}_{j}^{m}: 1 \leq j \leq n_{m}\right\}$ denote the open connected components of $\left\{x \in \mathbb{R}^{l}: h_{m}(x) \neq 0\right\}$ and let $T_{\bullet}^{m}:=\min \left(t>0: h_{m}(X(t))=0\right)$. The twisted generator $\check{D}_{i}$ is defined as before by a similarity transformation and a translation:

$$
\check{\mathscr{D}}_{i}=I_{h_{i}}^{-1} \mathscr{D} I_{h_{i}}+\Gamma_{i} I
$$

where $\Gamma_{i}:=\left|\Lambda_{i}\right|$ for $i \geq 2$.

For $i=2,3,4$ we may conclude from Theorem 3.7 that the associated twisted process on any $\mathrm{M}_{j}^{i}$ is $\check{V}_{i}$-uniformly ergodic with $h^{-1} \in L_{\infty}^{\check{V}_{i}}$. Consequently, each of these sets is metastable, with exit rate equal to $\Gamma_{i}, i=2,3$, and, moreover, from the definition of the twisted process,

$$
\mathrm{E}_{x}\left[\exp \left(\Gamma_{i} T_{\bullet}^{i}\right)\right]=\infty, \quad x \in \mathrm{M}_{j}^{i}, 1 \leq j \leq n_{i} .
$$

This is a dramatic statement since $\Gamma_{i}:=\left|\Lambda_{i}\right| \sim 0$ for $i \leq 3$. Similarly, for all $\Gamma<\Gamma_{4}$,

$$
\mathrm{E}_{x}\left[\exp \left(\Gamma T_{\bullet}^{4}\right)\right]<\infty, \quad x \in \mathrm{M}_{j}^{4}, 1 \leq j \leq n_{4},
$$

so that the process exits these sets relatively quickly.

It appears then that one should focus on the process with generator $\check{\mathscr{D}}_{3}$. If Figure 4 is accepted as an illustration of the spectrum of this generator (ignoring those eigenvalues in the right-half plane), it would then follow that the associated twisted process has a relatively large spectral gap. It is in this situation that the conclusions of Theorem 3.8 have the greatest impact.

Here we investigate a refinement of this decomposition using two eigenfunctions as follows: 
State space decompositions.

(i) The shattered state space $\mathrm{S} \subset \mathcal{C}$ is given by

$$
\mathrm{S}:=\left\{\begin{array}{c}
\text { connected components of the open set } \\
\left\{x \in \mathbb{X}: h_{2}(x) h_{3}(x) \neq 0\right\}
\end{array}\right\} .
$$

We denote by $a, b, c, \ldots$ generic elements of $S$ and we denote the exit time from any $s \in \mathrm{S}$ by

$$
T_{\bullet}:=\min \left(T_{\bullet}^{2}, T_{\bullet}^{3}\right) .
$$

(ii) Suppose that $\mathrm{a} \in \mathrm{S}$ with $h_{4}(x) \neq 0$ for all $x \in \mathrm{a}$. Then the set a is called a transition set.

We do not know if any of the sets in $S$ are metastable, although there are numerous combinations $\mathbf{M}=\mathrm{a}_{1} \circ \mathrm{a}_{2} \circ \cdots \circ \mathrm{a}_{k}$ that are metastable for $\mathbf{X}$. When a subset $\left\{\mathrm{a}_{i}: 1 \leq i \leq k\right\} \subset \mathrm{S}$ has this property it may be viewed as a metastable subchain of a finite state-space chain with alphabet $\mathrm{S}$. Lower bounds on exit rates, in particular the behavior of the process on a transition set, are addressed in the following proposition.

Proposition 4.1. For any $\mathrm{a} \in \mathrm{S}$ we have $\Gamma(\mathrm{a}) \geq \Gamma_{3}$. If a is a transition set we have $\Gamma(\mathrm{a}) \geq \Gamma_{4}$.

PROOF. The bound $\Gamma(\mathrm{a}) \geq \Gamma_{3}$ is obtained as follows: every $\mathrm{a} \in \mathrm{S}$ is contained in one of the sets $\mathrm{M}_{j}^{3}$, so we may assume that $h_{3}$ is strictly positive on a. Theorem 3.2(i) implies that $\Gamma$ (a) $\geq \Gamma_{3}$ since we also have $D h_{3}=-\Gamma_{3} h_{3}$ on a.

If a is a transition set, then $a \subset \mathrm{M}_{j}^{4}$ for some $j$. Identical reasoning implies that $\Gamma(\mathrm{a}) \geq \Gamma_{4}$.

Our goal is to build a finite state-space Markov chain on the finite alphabet $S$ and view major transitions of the diffusion as simple jumps of this Markov chain. We introduce some suggestive directions for future research here, but fall short of proving an exact correspondence between this chain and the diffusion. A precise approximation is possible by considering a sequence of processes whose spectral gap approaches infinity. We illustrate this in Section 5 through results from numerical experiments.

Given any decomposition of the state space $S=\left\{a_{1}, \ldots, a_{m}\right\} \subset \mathcal{C}$ we consider the following guidelines in the construction of a rate matrix $Q=\left(q_{i j}\right)$ to represent an approximating Markov chain. Properties (R2) and (R3) ensure that $Q$ generates stochastic matrices $e^{t Q}$ for all $t \geq 0$. 


\section{Conditions on rate matrix $Q$.}

(R1) Diagonal elements given by $q_{i i}=-\Gamma\left(\mathrm{a}_{i}\right)$.

(R2) $\sum_{j} q_{i j}=0$ for all $j$.

(R3) $q_{i j} \geq 0$ for $i \neq j$.

(R4) $Q$ generates a unique invariant measure, that is, the eigenspace of its largest eigenvalue 0 is one-dimensional.

For any finite decomposition a rate matrix satisfying these conditions may be defined as follows: The diagonal elements are given by (R1) and for any two neighbors $\mathrm{a}_{i}, \mathrm{a}_{j} \in \mathrm{S}$ we define

$$
q_{i, j}=p_{i} \Gamma\left(\mathrm{a}_{i} \mid \mathrm{a}_{i} \circ \mathrm{a}_{j}\right),
$$

where $p_{i}$ is the normalizing constant,

$$
p_{i}:=\Gamma\left(\mathrm{a}_{i}\right)\left[\sum\left\{\Gamma\left(\mathrm{a}_{i} \mid \mathrm{a}_{i} \circ \mathrm{a}_{k}\right): \mathrm{a}_{k} \text { is a neighbor of } \mathrm{a}_{i}\right\}\right]^{-1} .
$$

This is the appropriate representation in the (unrealistic) case, where

$$
\widehat{X}(t)=\left(\mathbf{1}_{\mathrm{a}_{1}}(X(t)), \ldots, \mathbf{1}_{\mathrm{a}_{m}}(X(t))\right), \quad t \in \mathbb{T},
$$

is a Markov chain. We next investigate how far the indicator process $\widehat{\mathbf{X}}$ deviates from a Markov chain.

4.3. Error bounds for Markov chain approximations. Suppose we are given $m$ disjoint sets $\left\{\mathrm{a}_{k}: k=1, \ldots, m\right\} \subset \mathcal{C}$ and assume that these shatter the state space in the sense that $\mathrm{a}_{1} \circ \cdots \circ \mathrm{a}_{m}=\mathbb{X}$. To construct an approximating Markov chain we consider the coupling matrix $W^{t}=\left(w_{k l}^{t}\right)_{k, l=1, \ldots, m}$ defined by the steady-state probabilities,

$$
W_{k l}^{t}=\mathrm{P}_{\pi}\left[X_{t} \in \mathrm{a}_{l} \mid X_{0} \in \mathrm{a}_{k}\right] .
$$

We assume that the semigroup is self-adjoint and compact so that there exists an orthonormal basis of eigenfunctions $\left\{h_{k}\right\}$ in $L_{\pi}^{2}$, where the dual product is given by

$$
\langle f, g\rangle_{\pi}:=\int_{\mathbb{X}} f(x) g(x) \pi(d x)
$$

and $\pi$ is the invariant measure of $\mathbf{X}$. This additional structure is convenient in obtaining simple bounds.

The normalized indicator functions of sets in $S$ are given by

$$
\chi_{k}=\mathbf{1}_{\mathrm{a}_{k}} / \sqrt{\pi\left(\mathrm{a}_{k}\right)}, \quad k=1, \ldots, m .
$$

This allows us to rewrite the matrix $\left\{W^{t}\right\}$ in the form

$$
\left(W^{t}\right)_{k l}=M^{-1 / 2} \cdot\left(\left\langle P^{t} \chi_{k}, \chi_{l}\right\rangle_{\pi}\right)_{k, l=1, \ldots, m} \cdot M^{1 / 2} .
$$


The $\left\{\chi_{k}\right\}$ may be expressed in terms of the eigenfunctions through the following expansion,

$$
\chi_{k}=\sum_{j=1}^{\infty} c_{k l} h_{l}, \quad \text { with } c_{k l}=\left\langle\chi_{k}, h_{l}\right\rangle_{\pi},
$$

which is convergent in $L^{2}(\pi)$. If we truncate this sum to $j \leq m$ we then obtain the projection of $\chi_{k}$ onto the subspace spanned by the first $m$ eigenfunctions. The mean-square error is given by

$$
\varepsilon_{k}^{2}:=\left\|\chi_{k}-\sum_{j=1}^{m} c_{k l} h_{l}\right\|_{\pi}^{2}=\sum_{j=m+1}^{\infty} c_{k l}^{2} .
$$

For any $t, k, l$ we have the representation,

$$
\begin{aligned}
\left\langle P^{t} \chi_{k}, \chi_{l}\right\rangle_{\pi} & =\sum_{j=1}^{\infty} c_{k i} e^{\Lambda_{j} t}\left\langle h_{i}, c_{l j} h_{j}\right\rangle_{\pi}=\sum_{j=1}^{\infty} c_{k j} e^{\Lambda_{j} t} c_{l j} \\
& =\left(\widehat{C} e^{\widehat{\Lambda} t} \widehat{C}^{T}\right)_{k l},
\end{aligned}
$$

with matrices $\widehat{C}=\left(c_{k j}\right) \in \mathbf{R}^{m \times \infty}$ and $\widehat{\Lambda}=\operatorname{diag}\left(\Lambda_{j}\right) \in \mathbf{R}^{\infty \times \infty}$. A truncation of this identity leads to a finite matrix with bounded error. Setting $s_{k}=\sum_{j=1}^{m} c_{k l} h_{l}$ and $e_{k}=\chi_{k}-s_{k}$ gives

$$
\left\langle P^{t} \chi_{k}, \chi_{l}\right\rangle_{\pi}=\left\langle P^{t} s_{k}, s_{l}\right\rangle_{\pi}+\left\langle P^{t} e_{k}, \chi_{l}\right\rangle_{\pi}+\left\langle P^{t} s_{k}, e_{l}\right\rangle_{\pi},
$$

with

$$
\begin{aligned}
\left|\left\langle P^{t} e_{k}, \chi_{l}\right\rangle_{\pi}\right| & \leq\left\|P^{t} e_{k}\right\|_{\pi} \cdot\left\|\chi_{l}\right\|_{\pi} \leq e^{\Lambda_{m+1} t} \varepsilon_{k}, \\
\left|\left\langle P^{t} s_{k}, e_{l}\right\rangle_{\pi}\right| & \leq\left\|P^{t} e_{l}\right\|_{\pi} \cdot\left\|s_{l}\right\|_{\pi} \leq e^{\Lambda_{m+1} t} \varepsilon_{l} \cdot\left(1+\varepsilon_{k}\right)
\end{aligned}
$$

and hence the bound,

$$
\left|\left\langle P^{t} \chi_{k}, \chi_{l}\right\rangle_{\pi}-\left\langle P^{t} s_{k}, s_{l}\right\rangle_{\pi}\right| \leq e^{\Lambda_{m+1} t}\left(\varepsilon_{k}+\varepsilon_{l}+\varepsilon_{k} \varepsilon_{l}\right) .
$$

Using these bounds we may compare the coupling matrix $\left\{W^{t}\right\}$ given in (28) with the semigroup for a finite state-space Markov chain. First observe that with

$$
C=\left(c_{k l}\right)_{k l=1, \ldots, m}, \quad D=\operatorname{diag}\left(e^{\Lambda_{1}}, \ldots, e^{\Lambda_{m}}\right) \quad \text { and } \quad M=\operatorname{diag}\left(\sqrt{\pi\left(\mathrm{a}_{k}\right)}\right),
$$

the previous definitions give

$$
\left(\left\langle P^{t} s_{k}, s_{l}\right\rangle_{\pi}\right)_{k, l=1, \ldots, m}=C D^{t} C^{T} .
$$

Setting

$$
\widehat{W}_{k l}^{t}:=\left(M^{-1 / 2} C D^{t} C^{T} M^{1 / 2}\right)_{k l},
$$


we henceforth get

$$
\begin{aligned}
\left\|W^{t}-\widehat{W}^{t}\right\|_{\pi} & \leq\left\|\left(\left\langle P^{t} \chi_{k}, \chi_{l}\right\rangle_{\pi}-\left\langle P^{t} s_{k}, s_{l}\right\rangle_{\pi}\right)_{k, l=1, \ldots, m}\right\|_{2} \\
& \leq m e^{\Lambda_{m+1} t} \cdot \underbrace{\max _{k, l}\left(\varepsilon_{k}+\varepsilon_{l}+\varepsilon_{k} \varepsilon_{l}\right)}_{\text {error indicator }} .
\end{aligned}
$$

In general the semigroup $\left\{\widehat{W}^{t}\right\}$ is not positive, though positivity is guaranteed if the approximation above is sufficiently tight.

5. Numerical example: the three-well potential. We conclude with some numerical results to better understand the "shattered state space," the finite statespace Markov chain and the exponential approximation of the exit times. We consider the Smoluchowski equation

$$
d X=-\frac{1}{\gamma} \nabla U(X) d t+\frac{\sigma}{\gamma} d W
$$

where the potential $U: \mathbb{R} \rightarrow \mathbb{R}_{+}$is smooth. The restriction to one-dimension is simply for ease of exposition and to avoid subtleties surrounding exotic stationary points for the potential $U$.

We have already remarked that this is an elliptic diffusion when $\sigma>0$. The diffusion $\mathbf{X}$ is ergodic provided the function $p_{0}(x):=\exp (-\kappa U(x))$ is integrable on $\mathbb{R}$, where $\kappa=2 \gamma / \sigma^{2}$ is the inverse temperature. In this case the invariant density for the stationary distribution is given by $p(x)=Z^{-1} p_{0}(x)$, where $Z$ is a normalizing constant. Under mild additional assumptions on the potential function $U$ one can verify that this Markov process is $V$-uniformly ergodic, with $V=e^{\varepsilon U}$ for some $\varepsilon>0[18,26]$.

For our numerical analysis we consider in greater detail the three-well potential introduced in the Introduction with

$$
U(x)=\frac{1}{200}\left(0.5 x^{6}-15 x^{4}+119 x^{2}+28 x+50\right) .
$$

The required eigenvalues and eigenvectors of the generator were computed numerically by means of finite element discretization with uniform grid in the interval $[-6,6]$, with piecewise linear ansatz functions and zero Dirichlet boundary conditions at $x= \pm 6.0$. Convergence and convergence rates of this procedure are known since the generator is self-adjoint in the Hilbert space $L_{\pi}^{2}$. The accuracy of the numerical approximations have been tested based on this supporting theory using grid refinement.

5.1. Exit rates and the shattered state space. Discretizing the generator corresponding to the three-well potential with parameters $\gamma=2.25, \sigma=1.5$ yields an inverse temperature $\kappa=2$ and the following spectrum:

\begin{tabular}{ccccccc}
\hline $\boldsymbol{\Lambda}_{\mathbf{1}}$ & $\boldsymbol{\Lambda}_{\mathbf{2}}$ & $\boldsymbol{\Lambda}_{\mathbf{3}}$ & $\boldsymbol{\Lambda}_{\mathbf{4}}$ & $\boldsymbol{\Lambda}_{\mathbf{5}}$ & $\boldsymbol{\Lambda}_{\mathbf{6}}$ & $\cdots$ \\
\hline 0.0000 & -0.0216 & -0.0381 & -0.4183 & -0.6509 & -0.9240 & $\ldots$ \\
\hline
\end{tabular}



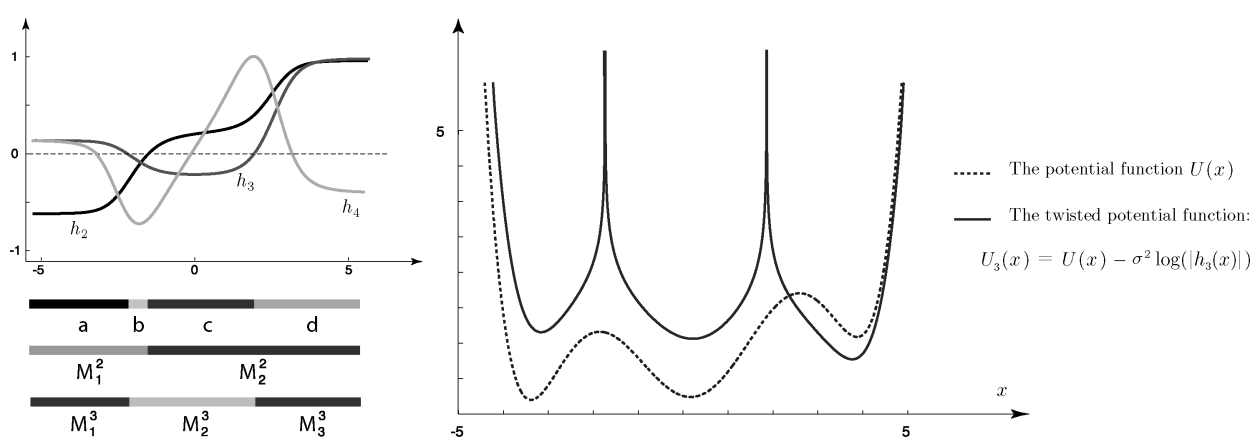

FIG. 5. On the left is shown the metastable sets and the shattered state space corresponding to the three-well potential for $\gamma=2.25$ and $\sigma=1.5$. At right is the potential $U_{3}(x)=U-\log \left(\left|h_{3}\right|\right)$ for the transformed generator (solid line).

The first four eigenfunctions are shown in Figure 5 for this value of $\kappa$.

For decreasing temperature, Figure 6 shows that the second and third eigenvalues converge to 0 , while the fourth and higher-order eigenvalues remain bounded from below with increasing $\kappa$. Here we consider decompositions of the state space when $\kappa=2$ and in Section 5.2 we consider in some detail the asymptotics of the eigensystem as $\kappa \rightarrow \infty$.

We may use the third eigenfunction $h_{3}$ to obtain the twisted process defined in Section 3.2. According to Proposition 3.4(iii), the twisted process is again a Smoluchowski equation corresponding to the potential function $U_{3}(x)=U(x)-$ $\sigma^{2} \log \left(\left|h_{3}(x)\right|\right)$, as shown in Figure 5, and with the same values of $\gamma$ and $\sigma$ as for the original Smoluchowski equation. Observe that the potential function $U_{3}$ is similar to the original potential function $U$ (dashed line), but the zeros of $h_{3}$
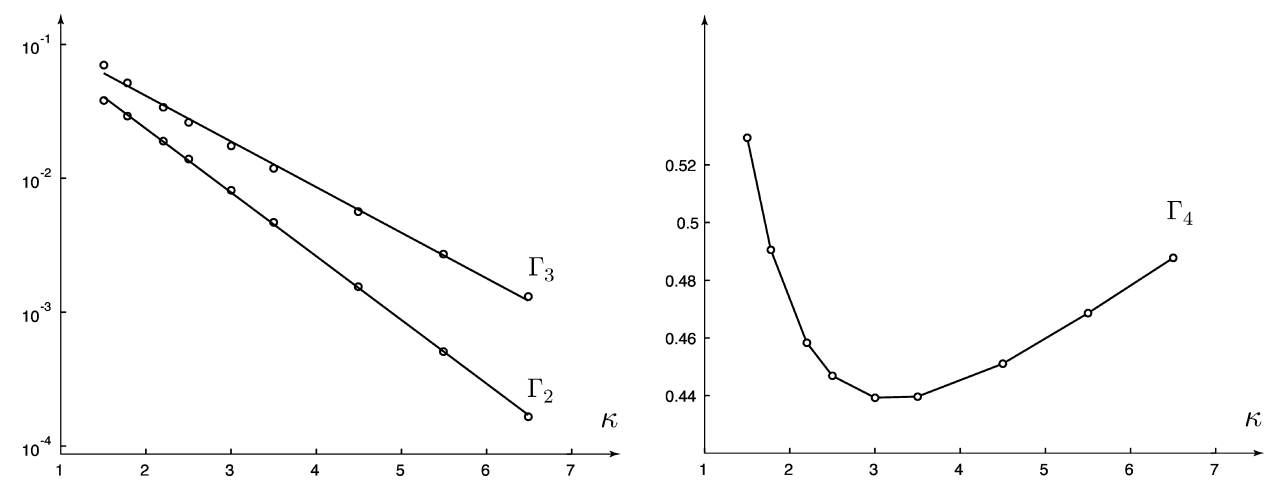

FIG. 6. Eigenvalues for the three-well potential as a function of $\kappa$. The second and third eigenvalue converge to zero (left), while (the magnitude of) the fourth eigenvalue is bounded away from zero (right). 
TABLE 1

\begin{tabular}{llc}
\hline Set s & $\Gamma(\mathbf{s})$ (theor.) & $\boldsymbol{\Gamma}(\mathbf{s})$ (exp.) \\
\hline $\mathrm{a}$ & $\Gamma_{3}=0.038$ & 0.036 \\
$\mathrm{~b}$ & $\Gamma(\mathrm{b})>\Gamma_{4}$ & 6.166 \\
$\mathrm{c}$ & $\Gamma(\mathrm{c})>\Gamma_{3}$ & 0.049 \\
$\mathrm{~d}$ & $\Gamma_{3}=0.038$ & 0.037 \\
$\mathrm{M}_{1}^{2}=\mathrm{a} \circ \mathrm{b}$ & $\Gamma_{2}=0.022$ & 0.021 \\
$\mathrm{M}_{2}^{2}=\mathrm{c} \circ \mathrm{d}$ & $\Gamma_{2}=0.022$ & 0.021 \\
$\mathrm{M}_{2}^{3}=\mathrm{b} \circ \mathrm{c}$ & $\Gamma_{3}=0.038$ & 0.035 \\
\hline
\end{tabular}

correspond to poles of $U_{3}$. This creates barriers in the state space for the twisted process, forming the decomposition $\mathbb{X}=\mathrm{M}_{1}^{3} \circ \mathrm{M}_{2}^{3} \circ \mathrm{M}_{3}^{3}$.

The shattered state space obtained using both eigenfunctions $h_{2}$ and $h_{3}$ consists of the four components $\mathrm{S}=\{\mathrm{a}, \mathrm{b}, \mathrm{c}, \mathrm{d}\}$ of the open set $\left\{x: h_{2}(x) h_{3}(x) \neq 0\right\}$, as shown at left in Figure 5. We have the following characterizations:

(i) The sets a and $d$ are two components of $\left\{h_{3}(x) \neq 0\right\}=\left\{M_{1}^{3}, M_{2}^{3}, M_{3}^{3}\right\}$. Due to Theorem 3.7 they are metastable with $\Gamma(\mathrm{a})=\Gamma(\mathrm{d})=\Gamma_{3}$.

(ii) The set $\mathrm{b}$ is a transition set since $h_{4}$ does not vanish on $\mathrm{b}$. Due to Proposition 4.1 we have $\Gamma(b)>\Gamma_{4}$.

(iii) The set $\mathrm{c}$ is a proper subset of the metastable set $\mathrm{M}_{2}^{3}$ and hence $\Gamma$ (c) > $\Gamma\left(\mathrm{M}_{2}^{3}\right)=\Gamma_{3}$ due to Theorem 3.7 and the definition of a metastable set on page 430 .

Numerical estimates of exit rates for the sets $\{a, b, c, d\}$ and their combinations are shown in Figure 7 when $\kappa=2$. These values were obtained by estimating the decay of the distribution of exit times for each set as follows: Given the initial state $x_{0}$ in the corresponding set, $N=120,000$ independent realizations of the diffusion process were simulated. Estimates of the exit time distribution for each set $s$ were obtained via detection of the exit time for each realization, from which the rate $\Gamma(\mathrm{s})$ is approximated by estimating the decay rate of the exponential tail through linear regression. Table 1 summarizes the results.

A more detailed investigation of the simulation data shows exactly how the exit time deviates from an exponential random variable. Recall that the conditional distribution function for the residual life at time $T \geq 0$ is given by

$$
F_{x}(s, T)=\mathrm{P}_{x}\left[\left(T_{\bullet}-T\right) \geq s \mid T_{\bullet} \geq T\right] .
$$

The plots shown in Figure 8 illustrate estimates of the residual life distribution function based on data obtained in the simulations described above.

The exit-time distribution shows two time-scale behavior when the initial condition $x_{0} \in \mathrm{s}$ is located near the boundary of the set $\mathrm{s}$ under consideration. 

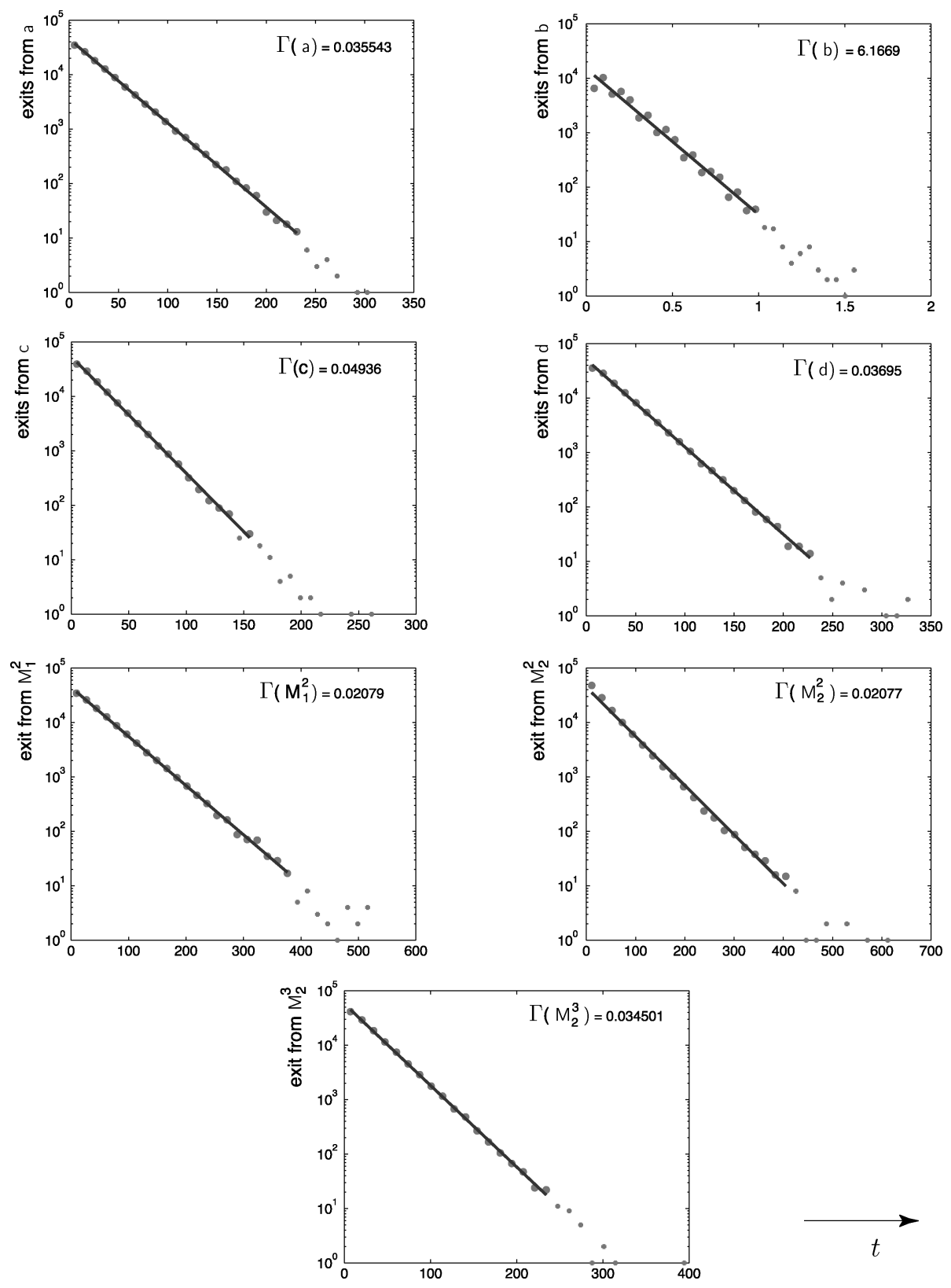

FIG. 7. Exit time statistics for the sets $\mathrm{S}=\{\mathrm{a}, \mathrm{b}, \mathrm{c}, \mathrm{d}\}$ and their combinations. Based on $N=120,000$ realizations, each figure shows a logarithmic plot of the number of realizations that have not exited up to the time specified on the horizontal axis. The decay rates of the distribution have been estimated via linear regression of the logarithmic data; only data with sufficient sampling information included (indicated with small circles). 

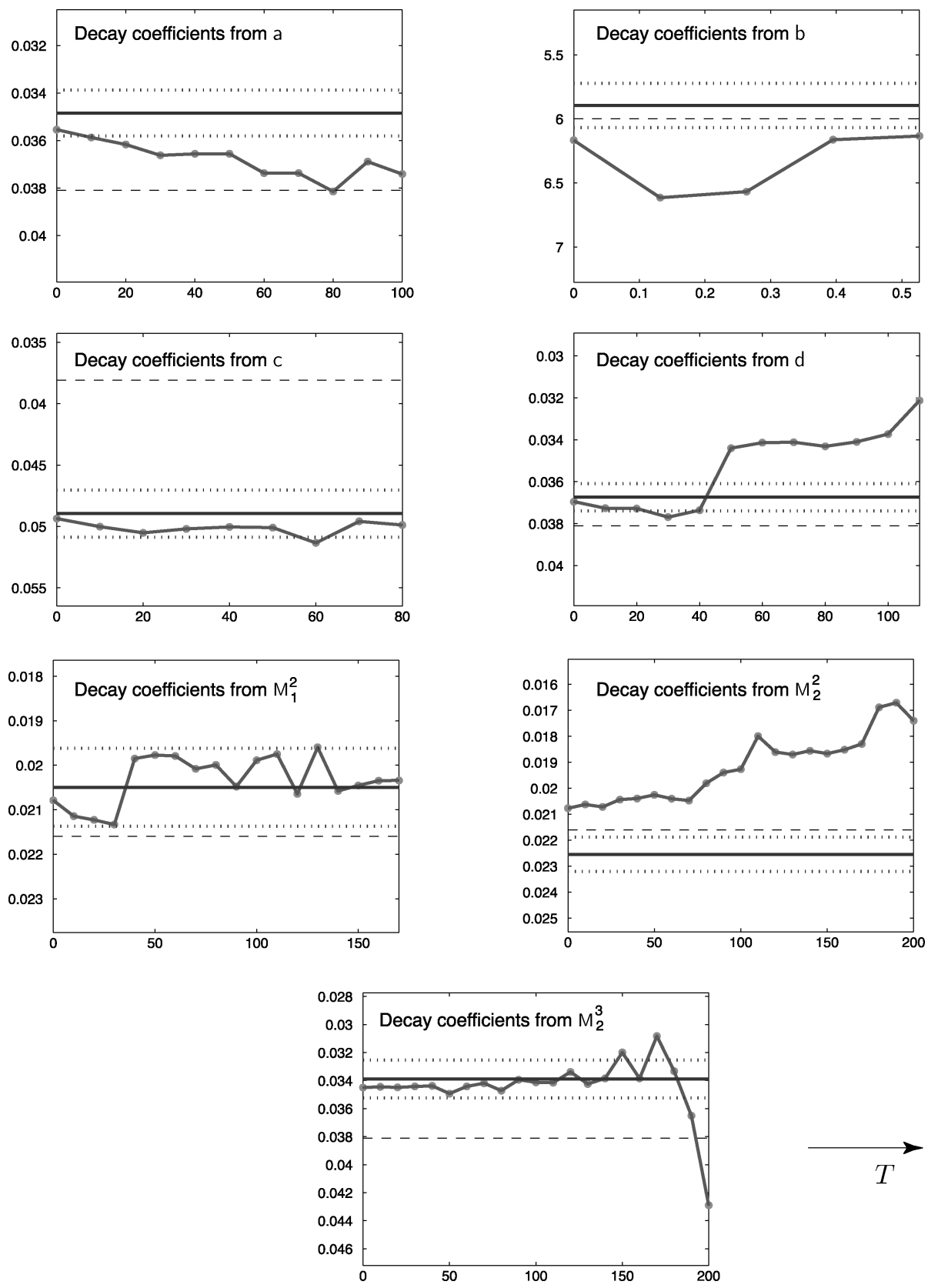

FIG. 8. Residual exit time distribution $F_{X}(\cdot, T)$ for the sets $\mathrm{S}$ and several combinations. The small circles indicate the estimates for the exponential decay rates of $F_{X}(\cdot, T)$ versus $T$ based on $N=120,000$ realizations. The solid and dotted lines indicate the average and variance of exit rates for 60 independent samples of length $N=2000$ each. The dashed line shows the theoretically expected value. 

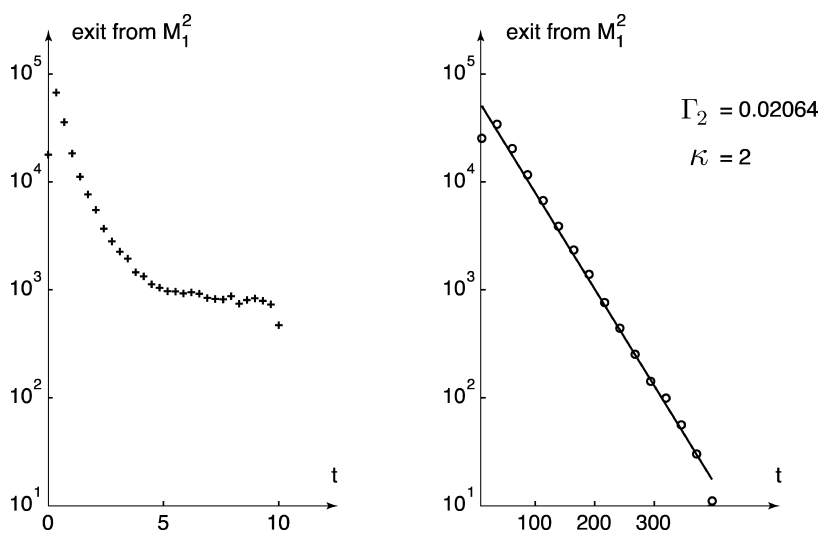

FIG. 9. Exit time statistics for the set $\mathrm{M}_{1}^{2}$. Based on $N=120,000$ realizations, each figure shows a logarithmic plot of the number of realizations that have not exited up to the time specified on the horizontal axis. The logarithmic plot after detailed regression exhibits two different decay rates. The tail of the distribution decays approximately due to $\Lambda_{2}$ (right), while initially (small exit times) the decay is substantially faster (left).

As shown in Figure 9, the residual life distribution possesses a high rate of decay initially and decays more slowly for higher values of the time $T$.

5.2. Asymptotic behavior of eigensystem and Markov chain approximations. We have already noted that the data shown in Figure 6 shows that the second and third eigenvalues converge to 0 exponentially fast as $\kappa \rightarrow \infty$, while the fourth eigenvalue, and therefore all remaining eigenvalues, do not vanish with increasing $\kappa$. If we consider decompositions of $\mathbb{X}=\mathbb{R}$ using $h_{2}$ or $h_{3}$, then we can predict the asymptotic values of their zeros by applying the implications from large deviations theory and Theorem 3.8 as stated at the end of Section 3.4. The results are illustrated in Figures 10 and 11.

We noted in Section 4.3 that an optimal representation based on an $L^{2}$ projection will give rise to an exact Markov chain model for vanishing temperature if there is a nonvanishing spectral gap beyond the first three eigenvalues. As the temperature decreases, the three-set representation $\left\{W^{t}\right\}$ of the diffusion process obtained in Section 4.3, equation (27), tends to a semigroup $\left\{\widehat{W}^{t}\right\}$ given by a rate matrix defined through (30). Figure 12 shows that the upper bound of the error indicator (31) tends to zero for vanishing temperature exponentially fast.

This is also illustrated in Figure 13. Recall that the normalized indicator functions $\left\{\chi_{i}\right\}$ given in (29) may be approximated by a linear combination of the first three eigenfunctions. As predicted in the discussion of Section 4.3, this approximation is increasingly accurate for increasing $\kappa$.

For large sampling times $t$ there is good agreement between $\left\{W^{t}\right\}$ and the semigroup $\left\{\widehat{W}^{t}\right\}$, even when the error indicator is large, since the pre-factor $e^{\Lambda_{m+1} t}$ 

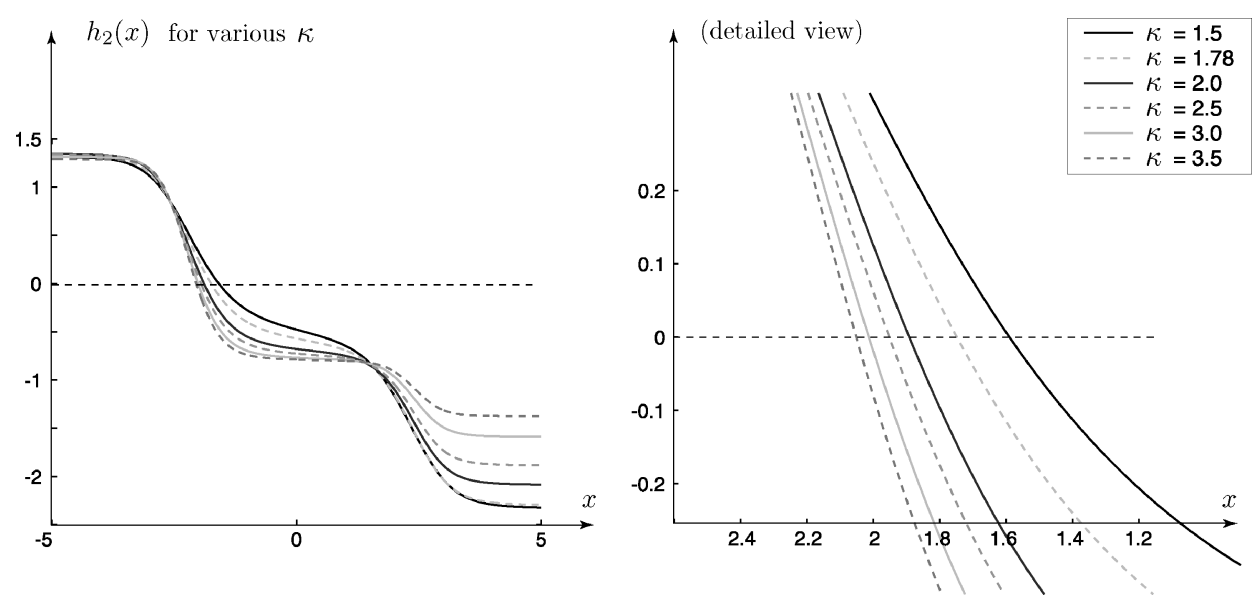

FIG. 10. The second eigenfunction for the three-well potential. Exactly as seen for the double-well potential, there exists a value $x_{21} \approx-2.42$ that breaks the state space into two regions with approximately equal exit rates. This point is asymptotically equal to the zero of the second eigenfunction. The right-hand side shows a close-up of the eigenfunctions shown at left.

of the upper bound (31) is small when there is a significant spectral gap. Figure 6 exhibits that $\Lambda_{m+1}=\Lambda_{4}$ is clearly bounded away from 0 for all values of $\kappa>0$.

In conclusion, we find that a four-state Markov chain does indeed accurately approximate the transition behavior of this diffusion, even for only moderately low temperature. In particular, for $\kappa=2$ the rate matrix $Q=\left(q_{i, j}\right)$ with $q_{i, j}=$ $p_{i} \Gamma\left(\mathrm{a}_{i} \mid \mathrm{a}_{i} \circ \mathrm{a}_{j}\right)$ defined in (27) is given by

$$
\begin{aligned}
& Q=\left(\begin{array}{cccc}
-\Gamma(\mathrm{a}) & \Gamma(\mathrm{a}) & 0 & 0 \\
p_{\mathrm{b}} \Gamma(\mathrm{b} \mid \mathrm{a} \circ \mathrm{b}) & -\Gamma(\mathrm{b}) & p_{\mathrm{b}} \Gamma(\mathrm{b} \mid \mathrm{c} \circ \mathrm{b}) & 0 \\
0 & p_{\mathrm{c}} \Gamma(\mathrm{c} \mid \mathrm{b} \circ \mathrm{c}) & -\Gamma(\mathrm{c}) & p_{\mathrm{c}} \Gamma(\mathrm{c} \mid \mathrm{d} \circ \mathrm{c}) \\
0 & 0 & \Gamma(\mathrm{d}) & -\Gamma(\mathrm{d})
\end{array}\right) \\
& \approx\left(\begin{array}{rrrr}
-0.036 & 0.036 & 0 & 0 \\
3.083 & -6.166 & 3.083 & 0 \\
0 & 0.016 & -0.049 & 0.033 \\
0 & 0 & 0.036 & -0.036
\end{array}\right)
\end{aligned}
$$

The eigenvalues of $Q$ are given by

$$
\begin{array}{llll}
\hline \Lambda_{1}(Q) & \Lambda_{2}(Q) & \Lambda_{3}(Q) & \Lambda_{4}(Q) \\
\hline 0.000 & -0.021 & -0.074 & -6.192 \\
\hline
\end{array}
$$

so that the second eigenvalue is nearly in agreement with $\Lambda_{2}=-0.022$ of the 

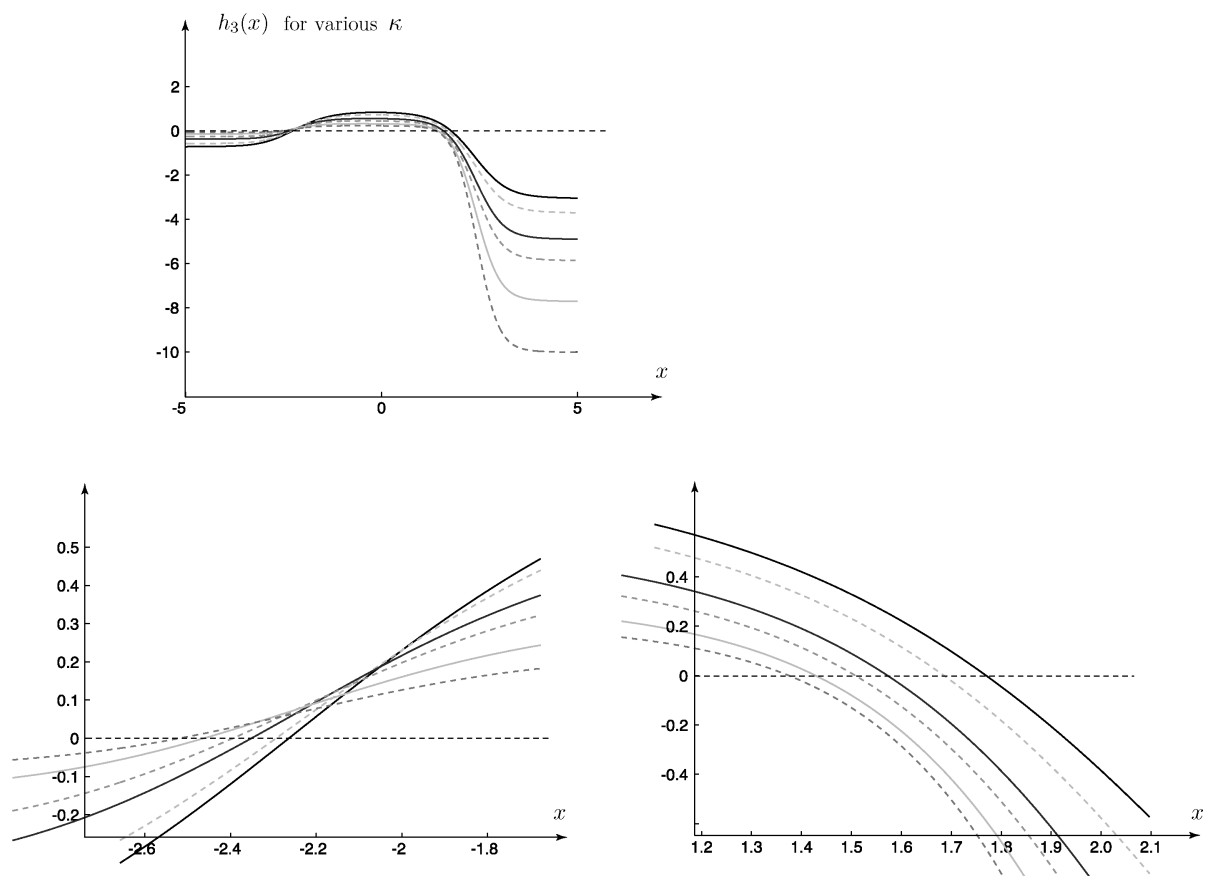

FIG. 11. The third eigenfunction, for various values of $\kappa$, together with two close-ups of the eigenfunction near its two zeros. We observe good convergence towards the predicted values $x_{31} \approx-3.11$ and $x_{32} \approx 1.37$.

diffusion [cf. (33)]. The second eigenvector of $Q$ is $v_{2}=(-0.870,-0.373,0.127$, $0.297)^{T}$, which also mimics the structure of the second eigenfunction $h_{2}$ (cf. Figure 5).

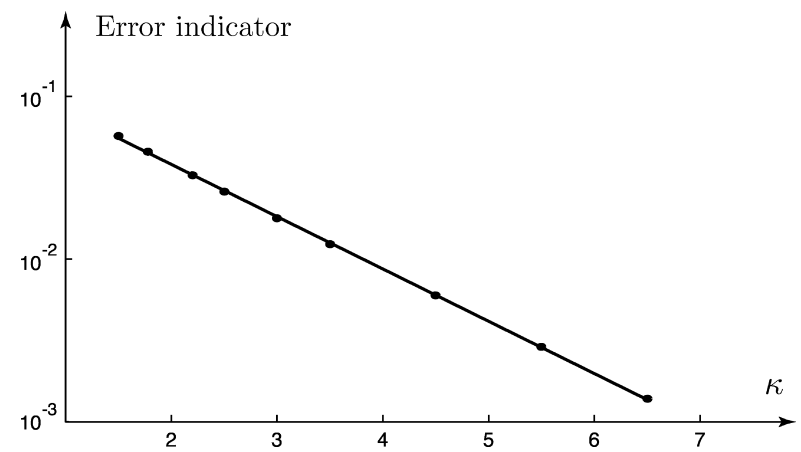

FIG. 12. Dependence of error indicator on $\kappa$ for the three-well potential. 

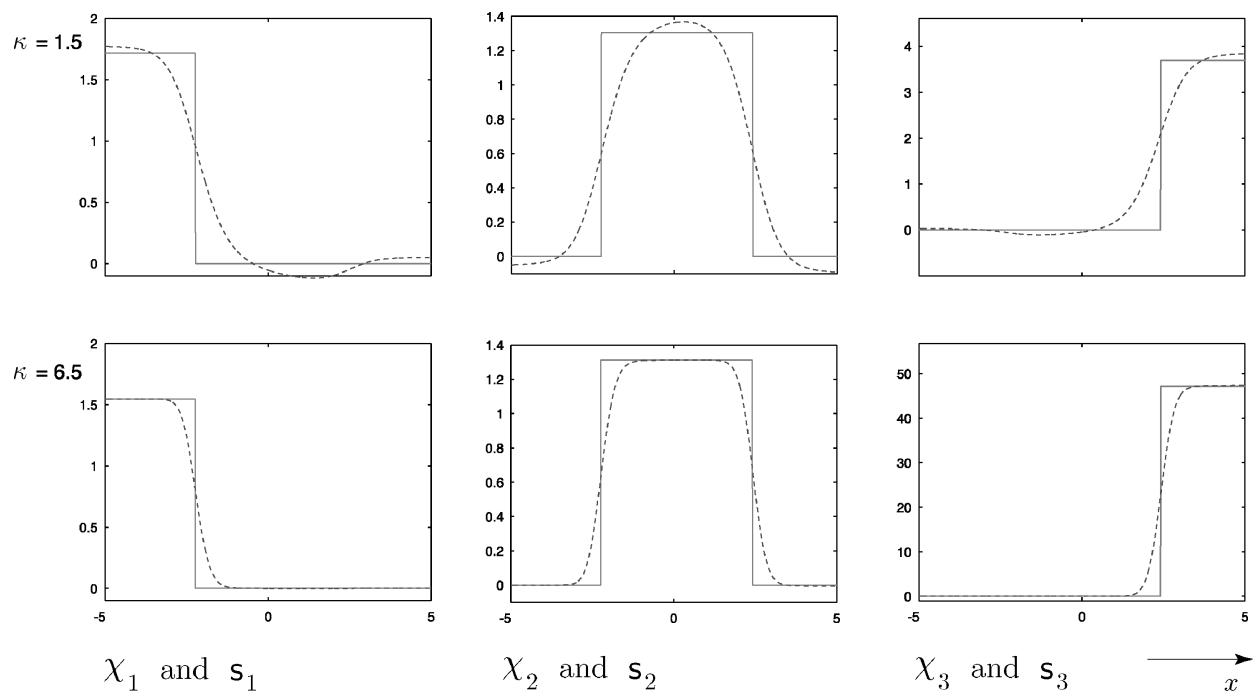

FIG. 13. Approximation of the normalized indicator functions $\chi_{i}$ by $s_{i}=$ optimal linear combinations of the first three eigenfunctions for the three-well potential. The three sets used to construct $\left\{\chi_{i}\right\}$ are designed based on the asymptotic location of the zeros according to Section 3.4. The top figure shows plots when $\kappa=1.5$, and in the lower figure $\kappa=6.5$. The approximation is far more accurate for larger values of $\kappa$, which is consistent with the data shown in Figure 12.

6. Outlook. In this paper we have developed some new tools for addressing the behavior of Markov processes restricted to a given domain and we have applied these methods to provide new bounds on the distribution of exit times. The numerical results suggest that these approximations are far more accurate than any computable bounds might reveal.

We are currently considering various extensions and refinements of these methods. In particular,

1. It may make no sense to search for eigenfunctions for high-dimensional models. In complex models we will require softer formulations of the eigenfunction equation (18), where $\Gamma_{0}$ is replaced by a function on $\mathrm{M}$. A twisted process can still be constructed, and analyzed according to the present paper, and this again gives bounds and statistical properties of exit times.

2. The approaches of [3] and [11] are based upon a variational representation of certain expectations, reminiscent of the variational representation of the rate function in large deviations theory (see [42]). We have recently shown that the large deviations rate function admits a variational representation as entropy for $V$-uniformly ergodic diffusions [20] and hope that some analogs will prove useful in providing a bridge between our methods and those in the references.

3. We have said nothing about the impact of a cluster of complex eigenvalues. It appears that a similar story may be told, but the constructed twisted process will be periodic in this case since metastable sets will exhibit a form of periodicity. 
4. It is a simple matter to show that the optimal representation obtained in Section 4.3 will approximate the diffusion by a finite state-space Markov chain under natural assumptions. What is less obvious is the accuracy of ad-hoc constructions such as (27). This is a topic of current research.

Acknowledgments. We are grateful to A. Bovier and I. Kontoyiannis for insightful discussions about exit times and large deviation theory, and several suggestions for improvements in the presentation.

\section{REFERENCES}

[1] Balaji, S. and Meyn, S. P. (2000). Multiplicative ergodicity and large deviations for an irreducible Markov chain. Stochastic Process. Appl. 90 123-144.

[2] Bolthausen, E., Deuschel, J.-D. and Tamura, Y. (1995). Laplace approximations for large deviations of nonreversible Markov processes. The nondegenerate case. Ann. Probab. 23 236-267.

[3] Bovier, A., Eckhoff, M., Gayrard, V. and Klein, M. (2001). Metastability in stochastic dynamics of disordered mean-field models. Probab. Theory Related Fields 119 99-161.

[4] Bovier, A., Eckhoff, M., Gayrard, V. and Klein, M. (2002). Metastability in reversible diffusion processes. I. Sharp asymptotics for capacities and exit times. Technical report.

[5] Bovier, A., Eckhoff, M., Gayrard, V. and Klein, M. (2002). Metastability in reversible diffusion processes. II. Precise asymptotics for small eigenvalues. Technical report.

[6] Bovier, A. and MAnzo, F. (2001). Metastability in Glauber dynamics in the low-temerature limit: Beyond exponential asymptotics. Preprint, Weierstrass-Institute für Angewandte Analysis und Stochastik.

[7] Deuflhard, P., Huisinga, W., Fischer, A. and SchÜtte, Ch. (2000). Identification of almost invariant aggregates in reversible nearly uncoupled Markov chains. Linear Algebra Appl. 315 39-59.

[8] Donsker, M. D. and Varadhan, S. R. S. (1983). Asymptotic evaluation of certain Markov process expectations for large time. IV. Comm. Pure Appl. Math. 36 183-212.

[9] Down, D., Meyn, S. P. and Tweedie, R. L. (1995). Exponential and uniform ergodicity of Markov processes. Ann. Probab. 23 1671-1691.

[10] FenG, J. and KuRTZ, T. G. (2000). Large deviations for stochastic processes. Preprint.

[11] Fleming, W. H. (1978). Exit probabilities and optimal stochastic control. Appl. Math. Optim. 4 329-346.

[12] Fleming, W. H. and James, M. R. (1992). Asymptotic series and exit time probabilities. Ann. Probab. 20 1369-1384.

[13] Fleming, W. H. and McEneaney, W. M. (1995). Risk-sensitive control on an infinite time horizon. SIAM J. Control Optim. 33 1881-1915.

[14] Fleming, W. H. and Sheu, S.-J. (1997). Asymptotics for the principal eigenvalue and eigenfunction of a nearly first-order operator with large potential. Ann. Probab. 25 1953-1994.

[15] Freidlin, M. I. and Wentzell, A. D. (1984). Random Perturbations of Dynamical Systems. Springer, New York.

[16] Glynn, P. W. and Thorisson, H. (2001). Two-sided taboo limits for Markov processes and associated perfect simulation. Stochastic Process. Appl. 91 1-20.

[17] Glynn, P. W. and ThORISSON, H. (2002). Structural characterization of taboo-stationarity for general processes in two-sided time. Stochastic Process. Appl. 102 311-318. 
[18] Huisinga, W. (2001). Metastability of Markovian systems: A transfer operator approach in application to molecular dynamics. Ph.D. thesis, Free Univ. Berlin.

[19] Jensen, J. L. (1987). A note on asymptotic expansions for Markov chains using operator theory. Adv. in Appl. Math. 8 377-392.

[20] Kontoyiannis, I. and MeYn, S. P. (2002). Large deviation asymptotics and the spectral theory of multiplicatively regular Markov processes. Submitted for publication.

[21] Kontoyiannis, I. and Meyn, S. P. (2003). Spectral theory and limit theorems for geometrically ergodic Markov processes. Ann. Appl. Probab. 13 304-362.

[22] KUnitA, H. (1978). Supports of diffusion processes and controllability problems. In Proceedings of the International Symposium on Stochastic Differential Equations (K. Itô, ed.) 163-185. Wiley, New York.

[23] Kunita, H. (1990). Stochastic Flows and Stochastic Differential Equations. Cambridge Univ. Press.

[24] Liberzon, D. and Brockett, R. W. (2000). Spectral analysis of Fokker-Planck and related operators arising from linear stochastic differential equations. SIAM J. Control Optim. 38 1453-1467.

[25] Lobry, C. (1970). Contrôlabilité des systèmes non linéaires. SIAM J. Control 8 573-605.

[26] Mattingly, J., Stuart, A. M. and Higham, D. J. (2001). Ergodicity for SDEs and approximations: Locally Lipschitz vector fields and degenerated noise. To appear.

[27] Meyn, S. P. and Tweedie, R. L. (1993). Generalized resolvents and Harris recurrence of Markov processes. Contemp. Math. 149 227-250.

[28] Meyn, S. P. and Tweedie, R. L. (1993). Markov Chains and Stochastic Stability. Springer, London.

[29] Meyn, S. P. and Tweedie, R. L. (1993). Stability of Markovian processes II: Continuous time processes and sampled chains. Adv. in Appl. Probab. 25 487-517.

[30] Neveu, J. (1964). Chaînes de Markov et théorie du potentiel. Ann. Fac. Sci. Univ. ClermontFerrand 24 37-89.

[31] Nummelin, E. (1984). General Irreducible Markov Chains and Nonnegative Operators. Cambridge Univ. Press.

[32] Rey-Bellet, L. and Thomas, L. E. (2000). Asymptotic behavior of thermal nonequilibrium steady states for a driven chain of anharmonic oscillators. Comm. Math. Phys. 215 1-24.

[33] Rey-Bellet, L. and Thomas, L. E. (2001). Fluctuations of the entropy production in anharmonic chains. To appear.

[34] Schütte, Ch., Fischer, A., Huisinga, W. and Deuflhard, P. (1999). A direct approach to conformational dynamics based on hybrid Monte Carlo. J. Comput. Phys. Special Issue on Computational Biophysics 151 146-168.

[35] SchÜtte, CH. and Huisinga, W. (2000). On conformational dynamics induced by Langevin processes. In EQUADIFF 99-International Conference on Differential Equations (B. Fiedler, K. Gröger and J. Sprekels, eds.) 2 1247-1262. World Scientific, Singapore.

[36] Schütte, Ch., Huisinga, W. and Deuflhard, P. (2001). Transfer operator approach to conformational dynamics in biomolecular systems. In Ergodic Theory, Analysis, and Efficient Simulation of Dynamical Systems (B. Fiedler, ed.). Springer, New York.

[37] Seneta, E. and Vere-Jones, D. (1966). On quasi-stationary distributions in discrete-time Markov chains with a denumerable infinity of states. J. Appl. Probab. $3403-434$.

[38] Stroock, D. W. and VARAdHAN, S. R. S. (1972). On the support of diffusion processes with applications to the strong maximum principle. Proc. Sixth Berkeley Symp. Math. Statist. Probab. 333-359. Univ. California Press, Berkeley.

[39] Sussmann, H. J. and Jurdjevic, V. (1972). Controllability of nonlinear systems. J. Differential Equations 12 95-116. 
[40] Thorisson, H. (2000). Coupling, Stationarity and Regeneration. Springer, New York.

[41] TweEdIE, R. L. (1974). Quasi-stationary distributions for Markov chains on a general state space. J. Appl. Probab. 11 726-741.

[42] VARAdHAN, S. R. S. (1984). Large Deviations and Applications. SIAM, Philadelphia, PA.

W. HUISINGA

C. SCHÜTTE

DEPARTMENT OF MATHEMATICS

AND COMPUTER SCIENCE

FREE UNIVERSITY BERLIN

GERMANY

E-MAIL: huisinga@math.fu-berlin.de schuette@math.fu-berlin.de
S. MEYN

DEPARTMENT OF ELECTRICAL AND COMPUTER ENGINEERING AND THE COORDINATED SCIENCES LABORATORY UNIVERSITY OF ILLINOIS AT URBANA-CHAMPAIGN URBANA, ILLINOIS 61801 USA

E-MAIL: s-meyn@uiuc.edu 